\title{
41. GRAIN-SIZE AND CARBON/CARBONATE ANALYSES, LEG 49
}

\author{
Stan M. White and Gerald W. Bode, Scripps Institution of Oceanography, La Jolla, California
}

\section{GRAIN-SIZE ANALYSES}

Sand-silt-clay distribution was determined on 10-cc sediment samples collected at the time the cores were split and described. The results are listed in Table 1.

The sediment classification used here is that of Shepard (1954) with the sand, silt, and clay boundaries based on the Wentworth (1922) scale (Figure 1). Thus the sand, silt, and clay fractions are composed of particles whose diameters range from 2000 to $62.5 \mu \mathrm{m}, 62.5$ to $3.91 \mu \mathrm{m}$, and less than $3.91 \mu \mathrm{m}$, respectively. This classification is applied regardless of sediment type and origin; therefore, the sediment names used in this table may differ from those used elsewhere in this volume; e.g., a silt composed of nannofossils in this table may be called a nannofossil ooze in a site chapter.

Standard sieve and pipette methods were used to determine the grain-size distribution. The sediment sample was dried and dispersed in a Calgon solution. If a sediment sample failed to disaggregate, it was treated with a sonic probe and, if necessary, hydrogen peroxide. Sediment samples which resisted the above treatment were not analyzed.

The sand fraction was removed by wet sieving, using a 63 $\mu \mathrm{m}$ sieve, and the silt and clay fractions were analyzed by standard pipette analysis. Sampling depths and times were calculated using equations derived from Stokes settling velocity equation (Krumbein and Pettijohn, 1938, p. 95-96):

$$
\begin{aligned}
\frac{D}{t^{*}} & =V=\frac{2 g r^{2}\left(d_{1}-d_{2}\right)}{9 \eta} \\
\mathrm{t} & =\frac{9 D \eta}{2 g r^{2}\left(d_{1}-d_{2}\right)}
\end{aligned}
$$

where

$V=$ velocity, in $\mathrm{cm} / \mathrm{sec}$

$t=$ time, in sec $*$

$D=$ depth pipette is inserted, in $\mathrm{cm}$

$g=$ gravity, in $\mathrm{cm} / \mathrm{sec}^{2 *}$

$r=$ radius of individual particles, in $\mathrm{cm}^{*}$

$d_{1}=$ density of solid particles arbitrarily set at $2.675 \mathrm{~g} / \mathrm{cc}$

$d_{2}=$ absolute density of distilled water at different temperatures (Hodgman et al., 1960, p. 2129)

$\eta=$ viscosity of distilled water in poises at different temperatures (Hodgman et al., 1960, p. 2181)

\footnotetext{
*Five figures were used in calculations to avoid rounding off variations.
}

The reproducibility of the grain size analysis has been previously tested (Boyce, 1972), and it was found that over a period of time with several operators the reproducibility for the sand-silt-clay fractions is $\pm 2.5 \%$ (absolute). For detailed step-by-step procedures, see Volume 4 of the Initial Reports of the Deep Sea Drilling Project.

\section{CARBON AND CARBONATE ANALYSES}

Leg 49 sediments were analyzed for total carbon and acid-insoluble (organic) carbon using the LECO WR-12 Analyzer according to the standard technique outlined below. The reproducibility of the LECO has been verified in extensive tests.

The $3-\mathrm{cm}^{3}$ sediment samples were first dried and ground into a homogenous powder. The ground sediment was redried at $105^{\circ}$ to $110^{\circ} \mathrm{C}$, and two samples, a $0.1-\mathrm{g}$ and a $0.5 \mathrm{~g}$ sample, were weighed into LECO clay crucibles. The 0.5 -g sample was acidified with 10 per cent hydrochloric acid and washed with distilled water. The sample was then dried and analyzed for acid-insoluble carbon. The 0.1-g sample was analyzed for total carbon without further treatment. If the sample contained less than 10 per cent $\mathrm{CaCO}_{3}$, an additional 0.5 -g sample was analyzed for greater accuracy. The calcium carbonate percentages were calculated as follows: (\% total C - \% organic C) $\times 8.33=\% \mathrm{CaCO}_{3}$. Although older carbonates may be present, all acid-soluble carbon was calculated as calcium carbonate. All results are given in weight percent (Table 2).

Detailed descriptions of the technique and theory may be found in Bader, Gerard, et al., (1970) and Boyce and Bode (1972).

\section{REFERENCES}

Bader, R. G., Gerard, R. D., et al., 1970. Initial Reports of the Deep Sea Drilling Project, v. 4: Washington (U.S. Government Printing Office).

Boyce, R. E., 1972. Grain size analyses, Leg 9, Deep Sea Drilling Project. In Hayes, J. D., Initial Reports of the Deep Sea Drilling Project, v. 9: Washington (U.S. Government Printing Office), p. 799.

Boyce, R.E., and Bode, G.W., 1972. Carbon and carbonate analyses, Leg 9, Deep Sea Drilling Project. In Hayes, J. D., et al., Initial Reports of the Deep Sea Drilling Project, v. 9; Washington (U.S. Government Printing Office), p. 747.

Hodgman, C. D., Weast, R. C. Y., and Selby, S. M., 1960. Handbook of Chemistry and Physics: Cleveland (Chemical Rubber Publishing Co.).

Krumbein, W. C. and Pettijohn, F. J., 1938. Manual of Sedimentary Petrography: New York (Appleton-CenturyCrofts, Inc.).

Shepard, F. P., 1954. Nomenclature based on sand-silt-clay ratios, J. Sediment. Petrol., v. 24, p. 151.

Wentworth, C. K., 1922. A scale of grade and class terms for clastic sediments, J. Geol., v. 30, p. 377. 
TABLE

Grain Size Analysis, Leg 49

\begin{tabular}{|c|c|c|c|c|c|}
\hline $\begin{array}{c}\text { Sample } \\
\text { (Interval in } \mathrm{cm} \text { ) }\end{array}$ & $\begin{array}{l}\text { Sub-Bottom } \\
\text { Depth }\end{array}$ & $\begin{array}{l}\text { Sand } \\
(\%)\end{array}$ & $\begin{array}{l}\text { Silt } \\
(\%)\end{array}$ & $\begin{array}{l}\text { Clay } \\
(\%)\end{array}$ & Classification \\
\hline \multicolumn{6}{|l|}{ Hole 407} \\
\hline $1-1,4$ & 0.04 & 32.4 & 30.5 & 37.0 & Sand-silt-clay \\
\hline $1-1,28$ & 0.28 & 22.2 & 38.3 & 39.6 & Sand-silt-clay \\
\hline $1-2,8$ & 1.58 & 30.1 & 33.4 & 36.6 & Sand-silt-clay \\
\hline $1-2,89$ & 2.39 & 30.9 & 37.4 & 31.7 & Sand-silt-clay \\
\hline $1-3,22$ & 3.22 & 17.2 & 40.7 & 42.1 & Silty clay \\
\hline $1-4,13$ & 4.63 & 30.6 & 43.3 & 26.1 & Sand-silt-clay \\
\hline $1-4,79$ & 5.29 & 23.9 & 43.2 & 32.9 & Sand-silt-clay \\
\hline $2-1,36$ & 6.36 & 20.0 & 33.5 & 46.5 & Silty clay \\
\hline $2-2,111$ & 8.61 & 56.8 & 23.1 & 20.1 & Sand-silt-clay \\
\hline $2-3,32$ & 9.32 & 45.3 & 20.8 & 33.9 & Sand-silt-clay \\
\hline $2-4,44$ & 10.94 & 25.5 & 30.1 & 44.4 & Sand-silt-clay \\
\hline $2-5,86$ & 12.86 & 17.9 & 41.1 & 41.0 & Clayey silt \\
\hline $2-6,46$ & 13.96 & 29.3 & 35.1 & 35.7 & Sand-silt-clay \\
\hline $3-1,34$ & 15.84 & 22.3 & 23.7 & 54.0 & Sand-silt-clay \\
\hline $3-2,107$ & 18.07 & 50.5 & 27.9 & 21.6 & Sand-silt-clay \\
\hline $3-4,21$ & 20.21 & 12.4 & 41.5 & 46.1 & Silty clay \\
\hline $4-1,104$ & 26.04 & 9.8 & 37.8 & 52.4 & Silty Clay \\
\hline $4-2,40$ & 26.90 & 14.4 & 45.8 & 39.8 & Clayey silt \\
\hline $4-3,92$ & 28.92 & 16.6 & 25.0 & 58.3 & Silty Clay \\
\hline $4-4,87$ & 30.37 & 12.3 & 41.1 & 46.6 & Silty clay \\
\hline $5-1,73$ & 35.23 & 22.7 & 35.2 & 42.1 & Sand-silt-clay \\
\hline $5-2,36$ & 36.36 & 23.9 & 42.7 & 33.5 & Sand-silt-clay \\
\hline $5-3,15$ & 37.65 & 30.5 & 43.4 & 26.0 & Sand-silt-clay \\
\hline $6-1,20$ & 44.20 & 9.7 & 39.4 & 51.0 & Silty clay \\
\hline $6-2,60$ & 46.10 & 3.3 & 56.8 & 40.0 & Clayey silt \\
\hline $6-3,67$ & 47.67 & 3.1 & 27.4 & 69.5 & Silty clay \\
\hline $7-1,90$ & 54.40 & 0.4 & 50.8 & 48.8 & Clayey silt \\
\hline $7-2,71$ & 55.71 & 6.6 & 52.7 & 40.8 & Clayey silt \\
\hline $8-2,70$ & 65.20 & 10.2 & 50.5 & 39.4 & Clayey silt \\
\hline $8-2,140$ & 65.90 & 7.1 & 54.0 & 38.9 & Clayey silt \\
\hline $8-3,15$ & 66.15 & 7.0 & 60.9 & 32.1 & Clayey silt \\
\hline $9-2,40$ & 74.40 & 3.2 & 51.1 & 45.7 & Clayey silt \\
\hline $10-1,90$ & 82.90 & 7.5 & 37.4 & 55.1 & Silty clay \\
\hline $10-2,40$ & 83.90 & 6.2 & 37.2 & 56.6 & Silty clay \\
\hline $10-3,89$ & 85.89 & 4.1 & 38.9 & 57.1 & Silty clay \\
\hline $10-4,23$ & 86.73 & 4.8 & 36.1 & 59.2 & Silty clay \\
\hline $11-1,60$ & 92.10 & 3.9 & 42.4 & 53.7 & Silty clay \\
\hline $11-2,60$ & 93.60 & 3.9 & 36.6 & 59.5 & Silty clay \\
\hline $11-3,60$ & 95.10 & 3.6 & 42.1 & 54.3 & Silty clay \\
\hline $11-4,60$ & 96.60 & 2.8 & 39.8 & 57.5 & Silty clay \\
\hline $12-1,74$ & 101.74 & 7.5 & 41.3 & 51.2 & Silty clay \\
\hline $12-2.74$ & 103.24 & 5.5 & 36.4 & 58.2 & Silty clay \\
\hline $12-3,75$ & 104.75 & 5.0 & 37.2 & 57.8 & Silty clay \\
\hline $13-1,85$ & 111.35 & 3.8 & 42.6 & 53.6 & Silty clay \\
\hline $13-2,83$ & 112.83 & 2.9 & 48.5 & 48.6 & Silty clay \\
\hline $13-3,83$ & 114.33 & 2.8 & 41.9 & 55.3 & Silty clay \\
\hline $13-4,83$ & 115.83 & 2.7 & 45.2 & 52.1 & Silty clay \\
\hline $13-5,55$ & 117.05 & 0.7 & 47.0 & 52.3 & Silty clay \\
\hline $13-6,55$ & 118.55 & 2.3 & 43.6 & 54.1 & Silty clay \\
\hline $14-1.74$ & 120.74 & 1.3 & 41.7 & 57.0 & Silty clay \\
\hline $14-2.74$ & 122.24 & 4.4 & 46.0 & 49.6 & Silty clay \\
\hline $14-3,74$ & 123.74 & 1.3 & 44.9 & 53.7 & Silty clay \\
\hline $14-4,29$ & 124.79 & 3.4 & 45.9 & 50.6 & Silty clay \\
\hline $16-1,82$ & 139.82 & 1.3 & 39.6 & 59.0 & Silty clay \\
\hline $16-2,82$ & 141.32 & 4.0 & 33.6 & 62.4 & Silty clay \\
\hline $16-3,82$ & 142.82 & 3.1 & 40.5 & 56.3 & Silty clay \\
\hline $16-4,10$ & 143.60 & 4.1 & 41.5 & 54.4 & Silty clay \\
\hline $17-1.78$ & 149.28 & 3.0 & 37.1 & 59.9 & Silty clay \\
\hline $17-2.78$ & 150.78 & 6.6 & 43.6 & 49.8 & Silty clay \\
\hline $17-3.78$ & 152.28 & 12.9 & 42.9 & 44.2 & Silty clay \\
\hline $18-1.75$ & 158.75 & 0.4 & 37.1 & 62.4 & Silty clay \\
\hline $18-2.75$ & 160.25 & 6.8 & 54.1 & 39.1 & Clayey silt \\
\hline $18-3.76$ & 161.76 & 9.0 & 52.3 & 38.7 & Clayey silt \\
\hline $18-4,76$ & 163.26 & 6.9 & 51.6 & 41.5 & Clayey silt \\
\hline $18-5.76$ & 164.76 & 8.1 & 54.8 & 37.1 & Clayey silt \\
\hline $19-1.76$ & 168.26 & 5.8 & 44.6 & 49.6 & Silty clay \\
\hline $19-2,76$ & 169.76 & 4.8 & 50.0 & 45.2 & (Clayey silt \\
\hline
\end{tabular}

TABLE 1 - Continued

\begin{tabular}{|c|c|c|c|c|c|}
\hline $\begin{array}{c}\text { Sample } \\
\text { (Interval in } \mathrm{cm} \text { ) }\end{array}$ & $\begin{array}{l}\text { Sub-Bottom } \\
\text { Depth }\end{array}$ & $\begin{array}{l}\text { Sand } \\
(\%)\end{array}$ & $\begin{array}{l}\text { Silt } \\
(\%)\end{array}$ & $\begin{array}{r}\text { Clay } \\
(\%)\end{array}$ & Classification \\
\hline $19-3,76$ & 171.26 & 5.2 & 45.4 & 49.3 & Silty clay \\
\hline $19-4,76$ & 172.76 & 10.5 & 52.3 & 37.1 & Clayey silt \\
\hline $19-5,76$ & 174.26 & 7.0 & 48.7 & 44.3 & Clayey silt \\
\hline $20-1,47$ & 177.47 & 1.2 & 44.9 & 53.8 & Silty clay \\
\hline $20-2,47$ & 178.97 & 0.3 & 32.9 & 66.8 & Silty clay \\
\hline $21-1,47$ & 186.97 & 1.0 & 44.9 & 54.0 & Silty clay \\
\hline $22-1,76$ & 196.76 & 5.2 & 47.2 & 47.6 & Silty clay \\
\hline $22-2,76$ & 198.26 & 1.9 & 47.7 & 50.3 & Silty clay \\
\hline $22-3,76$ & 199.76 & 1.4 & 45.8 & 52.8 & Silty clay \\
\hline $23-1,76$ & 206.26 & 1.4 & 32.7 & 65.9 & Silty clay \\
\hline $23-2,76$ & 207.76 & 2.2 & 42.0 & 55.8 & Silty clay \\
\hline $23-3,76$ & 209.26 & 0.7 & 41.2 & 58.1 & Silty clay \\
\hline $24-1,76$ & 215.76 & 4.0 & 52.7 & 43.3 & Clayey silt \\
\hline $24-2,76$ & 217.26 & 4.7 & 46.0 & 59.3 & Silty clay \\
\hline $24-3,76$ & 218.76 & 4.6 & 39.0 & 56.5 & Silty clay \\
\hline $24-4,76$ & 220.26 & 7.1 & 44.9 & 48.0 & Silty clay \\
\hline $24-6,76$ & 223.26 & 4.2 & 51.5 & 44.3 & Clayey silt \\
\hline $24-7,17$ & 224.17 & 2.4 & 48.0 & 49.6 & Silty clay \\
\hline $25-1,4$ & 224.54 & 1.4 & 39.9 & 58.7 & Silty clay \\
\hline $26-1,40$ & 234.40 & 2.6 & 40.2 & 57.2 & Silty clay \\
\hline $28-1,65$ & 253.65 & 11.8 & 46.6 & 41.6 & Clayey silt \\
\hline $28-2,65$ & 255.15 & 16.4 & 48.1 & 35.5 & Clayey silt \\
\hline $28-3,65$ & 256.65 & 9.1 & 55.4 & 35.5 & Clayey silt \\
\hline $28-4,65$ & 258.15 & 14.4 & 56.8 & 28.8 & Clayey silt \\
\hline $28-5,67$ & 259.67 & 5.3 & 50.6 & 44.0 & Clayey silt \\
\hline $29-1,60$ & 263.10 & 8.4 & 51.6 & 39.9 & Clayey silt \\
\hline $29-5,17$ & 268.67 & 13.8 & 48.7 & 37.5 & Clayey silt \\
\hline $30-1.69$ & 272.69 & 4.4 & 53.0 & 42.6 & Clayey silt \\
\hline $30-2,68$ & 274.18 & 3.7 & 51.2 & 45.1 & Clayey silt \\
\hline $30-3,59$ & 275.59 & 4.1 & 50.4 & 45.5 & Clayey silt \\
\hline $30-4,70$ & 277.20 & 2.8 & 51.3 & 45.9 & Clayey silt \\
\hline $30-5,71$ & 278.71 & 3.8 & 50.5 & 45.7 & Clayey silt \\
\hline $30-6,29$ & 279.79 & 2.5 & 41.2 & 56.3 & Silty clay \\
\hline $31-1,67$ & 282.17 & 7.4 & 47.3 & 45.2 & Clayey silt \\
\hline $31-2,67$ & 283.67 & 18.0 & 46.6 & 35.4 & Clayey silt \\
\hline $31-3,65$ & 285.15 & 10.4 & 46.2 & 43.3 & Clayey silt \\
\hline $31-4,67$ & 286.67 & 4.0 & 50.9 & 45.0 & Clayey silt \\
\hline $31-5,60$ & 288.10 & 9.6 & 45.6 & 44.8 & Clayey silt \\
\hline $31-6,67$ & 289.67 & 33.0 & 42.3 & 24.7 & Sand-silt-clay \\
\hline $31-7,20$ & 290.70 & 26.9 & 42.9 & 30.2 & Sand-silt-clay \\
\hline $32-1,27$ & 291.27 & 35.1 & 27.9 & 36.9 & Sand-silt-clay \\
\hline \multicolumn{6}{|l|}{ Hole 408} \\
\hline $1-1.60$ & 0.60 & 36.7 & 24.8 & 38.5 & Sand-silt-clay \\
\hline $1-2,19$ & 1.69 & 51.9 & 26.9 & 21.2 & Sand-silt-clay \\
\hline $1-3,29$ & 3.29 & 50.5 & 24.3 & 25.3 & Sand-silt-clay \\
\hline $1-4,82$ & 5.32 & 33.6 & 38.5 & 27.9 & Sand-silt-clay \\
\hline $1-5,41$ & 6.41 & 36.9 & 35.8 & 27.3 & Sand-silt-clay \\
\hline $1-6,22$ & 7.72 & 38.9 & 37.7 & 23.3 & Sand-silt-clay \\
\hline $2-1,99$ & 10.49 & 15.7 & 37.4 & 46.9 & Silty clay \\
\hline $2-2,10$ & 11.10 & 23.3 & 30.4 & 46.3 & Sand-silt-clay \\
\hline $2-3,69$ & 13.19 & 30.3 & 30.6 & 39.1 & Sand-silt-clay \\
\hline $2-4,30$ & 14.30 & 28.4 & 71.0 & 0.6 & Sandy silt \\
\hline $2-5,30$ & 15.80 & 55.6 & 23.3 & 21.1 & Sand-silt-clay \\
\hline $3-1.34$ & 19.34 & 22.1 & 43.1 & 34.8 & Sand-silt-clay \\
\hline $3-2,18$ & 20.68 & 36.3 & 35.5 & 28.2 & Sand-silt-clay \\
\hline $3-3,25$ & 22.25 & 31.9 & 32.1 & 36.0 & Sand-silt-clay \\
\hline $3-4,19$ & 23.69 & 11.9 & 44.9 & 43.2 & Clayey silt \\
\hline $3-5.6$ & 25.06 & 15.2 & 50.5 & 34.3 & Clayey silt \\
\hline $4-1,10$ & 28.60 & 30.6 & 37.1 & 32.3 & Sand-silt-clay \\
\hline $4-2,30$ & 30.30 & 23.0 & 46.9 & 30.0 & Sand-silt-clay \\
\hline $4-3,120$ & 32.70 & 11.7 & 47.6 & 4018 & Clayey silt \\
\hline $4-4,29$ & 33.29 & 39.4 & 31.7 & 28.9 & Sand-silt-clay \\
\hline $4-5,29$ & 34.79 & 21.5 & 50.1 & 28.5 & Sand-silt-clay \\
\hline $5-1,39$ & 38.39 & 12.8 & 46.7 & 40.5 & Clayey silt \\
\hline $5-3.54$ & 41.54 & 8.2 & 46.4 & 45.4 & Clayey silt \\
\hline $5-4,20$ & 42.70 & 4.5 & 23.0 & 52.5 & Silty clay \\
\hline $5-5,7$ & 44.07 & 6.2 & 60.9 & 33.0 & Clayey silt \\
\hline
\end{tabular}




\begin{tabular}{|c|c|c|c|c|c|}
\hline $\begin{array}{c}\text { Sample } \\
\text { (Interval in } \mathrm{cm} \text { ) }\end{array}$ & $\begin{array}{l}\text { Sub-Bottom } \\
\text { Depth }\end{array}$ & $\begin{array}{c}\text { Sand } \\
(\%)\end{array}$ & $\begin{array}{l}\text { Silt } \\
(\%)\end{array}$ & $\begin{array}{l}\text { Clay } \\
(\%)\end{array}$ & Classification \\
\hline $6-1,24$ & 47.74 & 11.5 & 48.0 & 40.6 & Clayey silt \\
\hline $6-2,39$ & 49.39 & 9.8 & 56.8 & 33.4 & Clayey silt \\
\hline $6-3,20$ & 50.70 & 7.4 & 58.0 & 34.6 & Clayey silt \\
\hline $7-1,30$ & 57.30 & 18.0 & 0.2 & 41.8 & Silty clay \\
\hline $7-2,6$ & 58.56 & 9.0 & 47.0 & 44.0 & Clayey silt \\
\hline $8-1,43$ & 66.93 & 5.2 & 40.1 & 54.7 & Silty clay \\
\hline $8-2,58$ & 68.58 & 7.5 & 43.1 & 49.3 & Silty clay \\
\hline $9-1,46$ & 76.46 & 5.8 & 38.9 & 55.3 & Silty clay \\
\hline $9-2,46$ & 77.96 & 5.8 & 45.8 & 48.4 & Silty clay \\
\hline $10-1,56$ & 86.06 & 6.6 & 17.1 & 76.2 & Clay \\
\hline $10-2,56$ & 87.56 & 9.6 & 47.8 & 42.5 & Clayey silt \\
\hline $10-3,56$ & 89.06 & 6.8 & 44.4 & 48.8 & Silty clay \\
\hline $10-4,56$ & 90.56 & 4.5 & 48.0 & 47.5 & Clayey silt \\
\hline $10-5,26$ & 91.76 & 4.9 & 39.3 & 55.8 & Silty clay \\
\hline $11-1,56$ & 95.56 & 6.3 & 45.0 & 48.7 & Silty clay \\
\hline $11-2,56$ & 97.05 & 2.2 & 55.8 & 42.0 & Clayey silt \\
\hline $11-3,56$ & 98.56 & 5.7 & 44.0 & 50.4 & Silty clay \\
\hline $11-4,56$ & 100.06 & 3.9 & 42.7 & 53.3 & Silty clay \\
\hline $11-5,56$ & 101.56 & 6.2 & 40.5 & 53.3 & Silty clay \\
\hline $11-6,36$ & 102.86 & 3.5 & 50.5 & 46.0 & Clayey silt \\
\hline $12-1,56$ & 105.06 & 4.3 & 46.9 & 48.8 & Silty clay \\
\hline $12-2,56$ & 106.56 & 7.3 & 44.0 & 48.6 & Silty clay \\
\hline $13-1,56$ & 114.56 & 6.5 & 43.2 & 50.3 & Silty clay \\
\hline $13-2,56$ & 116.06 & 3.0 & 41.9 & 55.1 & Silty clay \\
\hline $13-3,56$ & 117.56 & 3.1 & 39.6 & 57.3 & Silty clay \\
\hline $15-1,56$ & 133.56 & 5.2 & 49.5 & 45.2 & Clayey silt \\
\hline $15-2,96$ & 135.46 & 4.5 & 56.6 & 38.9 & Clayey silt \\
\hline $15-3,56$ & 136.56 & 3.2 & 52.5 & 44.2 & Clayey silt \\
\hline $15-4,56$ & 138.06 & 3.8 & 43.0 & 53.2 & Silty clay \\
\hline $15-5,56$ & 139.56 & 5.1 & 45.5 & 49.4 & Silty clay \\
\hline $16-1,106$ & 143.56 & 2.5 & 35.7 & 61.8 & Silty clay \\
\hline $16-2,56$ & 144.56 & 3.1 & 40.6 & 56.4 & Silty clay \\
\hline $16-3,26$ & 145.76 & 5.5 & 34.8 & 59.7 & Silty clay \\
\hline $17-1,54$ & 152.54 & 20.8 & 37.3 & 41.9 & Sand-silt-clay \\
\hline $17-2,54$ & 154.04 & 16.9 & 36.8 & 46.2 & Silty clay \\
\hline $17-3,56$ & 155.56 & 12.6 & 36.3 & 51.1 & Silty clay \\
\hline $17-4,56$ & 157.06 & 16.0 & 32.4 & 51.6 & Silty clay \\
\hline $17-5,56$ & 158.56 & 21.3 & 37.4 & 41.3 & Sand-silt-clay \\
\hline $18-1,106$ & 162.56 & 14.5 & 45.3 & 40.2 & Clayey silt \\
\hline $18-2,26$ & 163.26 & 8.6 & 40.8 & 50.6 & Silty clay \\
\hline $18-3,56$ & 165.06 & 12.8 & 45.2 & 42.0 & Clayey silt \\
\hline $18-4,16$ & 166.16 & 6.9 & 44.0 & 49.1 & Silty clay \\
\hline $19-1,26$ & 171.26 & 14.9 & 37.2 & 48.0 & Silty clay \\
\hline $19-2,56$ & 173.06 & 8.9 & 36.4 & 54.7 & Silty clay \\
\hline $19-3,56$ & 174.56 & 8.6 & 32.7 & 58.7 & Silty clay \\
\hline $19-4,56$ & 176106 & 8.4 & 37.9 & 53.6 & Silty clay \\
\hline $19-5,56$ & 177.56 & 5.6 & 38.0 & 56.4 & Silty clay \\
\hline $19-6,56$ & 179.06 & 7.1 & 46.4 & 46.6 & Silty clay \\
\hline $19-7,16$ & 180.16 & 12.0 & 36.2 & 51.7 & Silty clay \\
\hline $20-1,56$ & 181.06 & 11.1 & 34.6 & 54.3 & Silty clay \\
\hline $20-2,56$ & 182.56 & 9.1 & 30.6 & 60.3 & Silty clay \\
\hline $21-1,56$ & 190.56 & 5.0 & 45.4 & 49.6 & Silty clay \\
\hline $21-2,56$ & 192.06 & 5.1 & 45.7 & 49.2 & Silty clay \\
\hline $21-3,56$ & 193.56 & 4.6 & 43.9 & 51.5 & Silty clay \\
\hline $21-4,56$ & 195.06 & 6.1 & 47.8 & 46.1 & Clayey silt \\
\hline $22-1,21$ & 199.71 & 3.7 & 39.2 & 57.0 & Silty clay \\
\hline $22-2,21$ & 201.21 & 7.5 & 39.9 & 53.5 & Silty clay \\
\hline $22-3,30$ & 202.80 & 5.2 & 36.0 & 58.8 & Silty clay \\
\hline $23-1,40$ & 209.40 & 4.4 & 36.2 & 59.3 & Silty clay \\
\hline $23-2,34$ & 210.84 & 7.4 & 37.2 & 55.4 & Silty clay \\
\hline $23-3,16$ & 212.16 & 7.8 & 39.8 & 52.3 & Silty clay \\
\hline $24-1,28$ & 218.78 & 7.9 & 38.1 & 54.1 & Silty clay \\
\hline $24-2,28$ & 220.28 & 10.2 & 41.1 & 48.6 & Silty clay \\
\hline $24-3,11$ & 221.61 & 7.7 & 39.2 & 53.1 & Silty clay \\
\hline $24-5,19$ & 224.69 & 6.3 & 40.9 & 52.7 & Silty clay \\
\hline $24-6,20$ & 226.20 & 3.5 & 38.2 & 58.3 & Silty clay \\
\hline $25-1,19$ & 228.19 & 5.3 & 27.3 & 67.4 & Silty clay \\
\hline $25-2,18$ & 229.68 & 3.0 & 24.4 & 72.6 & Silty clay \\
\hline
\end{tabular}

\begin{tabular}{|c|c|c|c|c|c|}
\hline $\begin{array}{c}\text { Sample } \\
\text { (Interval in } \mathrm{cm} \text { ) }\end{array}$ & $\begin{array}{l}\text { Sub-Bottom } \\
\text { Depth }\end{array}$ & $\begin{array}{l}\text { Sand } \\
(\%)\end{array}$ & $\begin{array}{l}\text { Silt } \\
(\%)\end{array}$ & $\begin{array}{r}\text { Clay } \\
(\%)\end{array}$ & Classification \\
\hline $25-3,20$ & 231.20 & 4.7 & 28.2 & 67.1 & Silty clay \\
\hline $25-4,35$ & 232.85 & 3.2 & 31.3 & 65.4 & Silty clay \\
\hline $25-5,29$ & 234.29 & 4.8 & 26.4 & 68.8 & Silty clay \\
\hline $25-6,32$ & 235.82 & 3.9 & 35.1 & 61.0 & Silty clay \\
\hline $26-1,35$ & 237.85 & 4.1 & 29.9 & 66.0 & Silty clay \\
\hline $26-2,30$ & 239.30 & 5.9 & 31.7 & 62.5 & Silty clay \\
\hline $26-3,30$ & 240.80 & 5.0 & 33.2 & 61.8 & Silty clay \\
\hline $26-4,30$ & 242.30 & 7.6 & 36.1 & 56.3 & Silty clay \\
\hline $26-5,30$ & 243.80 & 8.4 & 40.0 & 51.6 & Silty clay \\
\hline $27-1,30$ & 247.30 & 12.7 & 40.1 & 47.2 & Silty clay \\
\hline $27-2,30$ & 248.80 & 5.2 & 28.6 & 66.2 & Silty clay \\
\hline $27-3,30$ & 250.30 & 8.2 & 35.6 & 56.2 & Silty clay \\
\hline $27-4,30$ & 251.80 & 15.6 & 34.6 & 49.7 & Silty clay \\
\hline $27-5,30$ & 253.30 & 28.1 & 31.6 & 40.3 & Sand-silt-clay \\
\hline $27-6,30$ & 254.80 & 12.3 & 36.8 & 50.9 & Silty clay \\
\hline $28-1,30$ & 256.80 & 7.0 & 44.0 & 49.0 & Silty clay \\
\hline $28-2,30$ & 258.30 & 12.7 & 36.9 & 50.3 & Silty clay \\
\hline $28-3,30$ & 259.80 & 6.6 & 42.2 & 51.2 & Silty clay \\
\hline $29-1,30$ & 266.30 & 5.0 & 35.0 & 60.0 & Silty clay \\
\hline $29-2,30$ & 267.80 & 2.9 & 35.7 & 61.5 & Silty clay \\
\hline $30-1,56$ & 276.06 & 14.3 & 39.5 & 46.2 & Silty clay \\
\hline $30-2,56$ & 277.56 & 15.8 & 43.8 & 40.3 & Clayey silt \\
\hline $30-3,56$ & 279.06 & 18.0 & 39.7 & 42.3 & Silty clay \\
\hline $30-4,56$ & 280.56 & 4.8 & 35.3 & 59.9 & Silty clay \\
\hline $30-5,56$ & 282.06 & 4.5 & 35.0 & 60.5 & Silty clay \\
\hline $31-1,56$ & 285.56 & 50.8 & 32.1 & 17.1 & Silty sand \\
\hline $31-2,56$ & 287.06 & 32.5 & 46.0 & 21.5 & Sand-silt-clay \\
\hline $31-3,56$ & 288.56 & 37.4 & 40.5 & 22.1 & Sand-silt-clay \\
\hline $31-4,56$ & 290.06 & 39.2 & 39.9 & 20.9 & Sand-silt-clay \\
\hline $31-5,56$ & 291.56 & 31.3 & 43.9 & 24.8 & Sand-silt-clay \\
\hline $31-6,56$ & 293.06 & 33.1 & 41.6 & 25.3 & Sand-silt-clay \\
\hline $32-1,56$ & 295.06 & 36.4 & 35.7 & 28.0 & Sand-silt-clay \\
\hline $32-2,56$ & 296.56 & 23.6 & 41.5 & 34.9 & Sand-silt-clay \\
\hline $32-3,56$ & 298.06 & 28.9 & 39.6 & 31.4 & Sand-silt-clay \\
\hline $32-4,56$ & 299.56 & 38.6 & 35.9 & 25.5 & Sand-silt-clay \\
\hline $32-5,56$ & 301.06 & 20.0 & 55.2 & 24.8 & Clayey silt \\
\hline $32-6,56$ & 302.56 & 19.6 & 59.6 & 20.9 & Clayey silt \\
\hline $33-1,56$ & 304.56 & 37.7 & 41.5 & 20.8 & Sand-silt-clay \\
\hline $33-2,56$ & 306.06 & 35.5 & 37.5 & 27.0 & Sand-silt-clay \\
\hline $33-3,56$ & 307.56 & 28.7 & 43.5 & 27.8 & Sand-silt-clay \\
\hline $33-4,56$ & 309.06 & 29.3 & 38.3 & 32.3 & Sand-silt-clay \\
\hline $33-5,56$ & 310.56 & 35.8 & 36.1 & 28.1 & Sand-silt-clay \\
\hline $33-6,56$ & 312.06 & 26.5 & 38.8 & 34.7 & Sand-silt-clay \\
\hline $34-1,56$ & 314.06 & 20.9 & 38.3 & 40.8 & Sand-silt-clay \\
\hline $34-2,56$ & 315.56 & 21.0 & 37.2 & 41.8 & Sand-silt-clay \\
\hline $34-3,56$ & 317.06 & 24.7 & 25.4 & 50.0 & Sand-silt-clay \\
\hline $34-4,56$ & 318.56 & 15.6 & 46.0 & 38.4 & Clayey silt \\
\hline $34-5,56$ & 320.06 & 3.9 & 57.2 & 38.9 & Clayey silt \\
\hline \multicolumn{6}{|l|}{ Hole 409} \\
\hline $2-1,56$ & 25.06 & 45.8 & 33.5 & 20.7 & Sand-silt-clay \\
\hline $2-2,56$ & 26.56 & 15.2 & 29.3 & 55.4 & Silty clay \\
\hline $2-3,56$ & 28.06 & 41.6 & 31.5 & 26.9 & Sand-silt-clay \\
\hline $4-1,56$ & 44.06 & 12.2 & 37.4 & 50.4 & Silty clay \\
\hline $4-2,56$ & 45.56 & 31.8 & 39.5 & 28.7 & Sand-silt-clay \\
\hline $4-3,56$ & 47.06 & 41.2 & 36.9 & 22.0 & Sand-silt-clay \\
\hline $4-4,56$ & 48.56 & 39.2 & 30.2 & 30.5 & Sand-silt-clay \\
\hline $4-5,8$ & 49.58 & 34.2 & 42.2 & 23.5 & Sand-silt-clay \\
\hline $5-1,56$ & 53.56 & 36.2 & 38.2 & 25.6 & Sand-silt-clay \\
\hline $5-2,56$ & 55.06 & 59.2 & 27.8 & 13.0 & Silty sand \\
\hline $5-3,56$ & 56.56 & 46.0 & 34.1 & 20.0 & Silty sand \\
\hline $5-4,66$ & 58.16 & 59.7 & 22.1 & 18.1 & Silty sand \\
\hline $6-1,68$ & 63.18 & 17.1 & 48.9 & 33.9 & Clayey silt \\
\hline $6-2,79$ & 64.79 & 23.4 & 45.2 & 31.4 & Sand-silt-clay \\
\hline $6-3,70$ & 66.20 & 5.9 & 48.6 & 45.5 & Clayey silt \\
\hline $7-1,79$ & 72.79 & 19.9 & 45.4 & 34.8 & Clayey silt \\
\hline $7-2,45$ & 73.95 & 35.4 & 34.4 & 30.2 & Sand-silt-clay \\
\hline $7-3,130$ & 76.30 & 10.5 & 51.0 & 38.5 & Clayey silt \\
\hline
\end{tabular}


TABLE 1 - Continued

\begin{tabular}{|c|c|c|c|c|c|}
\hline $\begin{array}{c}\text { Sample } \\
\text { (Interval in } \mathrm{cm} \text { ) }\end{array}$ & $\begin{array}{l}\text { Sub-Bottom } \\
\text { Depth }\end{array}$ & $\begin{array}{l}\text { Sand } \\
(\%)\end{array}$ & $\begin{array}{l}\text { Silt } \\
(\%)\end{array}$ & $\begin{array}{l}\text { Clay } \\
(\%)\end{array}$ & Classification \\
\hline $7-3,130$ & 76.30 & 10.5 & 51.0 & 38.5 & Clayey silt \\
\hline $7-4,89$ & 77.39 & 29.6 & 40.3 & 30.1 & Sand-silt-clay \\
\hline $7-5,82$ & 78.82 & 37.9 & 38.4 & 23.7 & Sand-silt-clay \\
\hline $7-6,35$ & 79.85 & 29.7 & 42.2 & 28.2 & Sand-silt-clay \\
\hline \multicolumn{6}{|l|}{ Hole 410} \\
\hline $1-1,30$ & 0.30 & 16.5 & 22.6 & 60.9 & Silty clay \\
\hline $1-2,28$ & 1.78 & 12.7 & 35.2 & 52.1 & Silty clay \\
\hline $1-3,28$ & 3.28 & 14.5 & 19.2 & 66.3 & Silty clay \\
\hline $1-4,40$ & 4.90 & 15.3 & 33.4 & 51.3 & Silty clay \\
\hline $1-5,38$ & 6.38 & 14.4 & 34.4 & 51.2 & Silty clay \\
\hline $2-1,58$ & 8.08 & 9.1 & 23.0 & 67.9 & Silty clay \\
\hline $2-2,40$ & 9.40 & 18.1 & 29.8 & 52.1 & Silty clay \\
\hline $2 \cdot 3,94$ & 11.44 & 5.6 & 17.7 & 76.6 & Clay \\
\hline $2-4,28$ & 12.28 & 11.1 & 16.3 & 72.7 & Silty clay \\
\hline $3-1,120$ & 18.20 & 7.2 & 18.4 & 74.4 & Silty clay \\
\hline $3-2,30$ & 18.80 & 6.5 & 12.6 & 80.9 & Clay \\
\hline $3-3,30$ & 20.30 & 11.3 & 12.2 & 76.5 & Clay \\
\hline $3-4,30$ & 21.80 & 16.8 & 24.7 & 58.5 & Silty clay \\
\hline $4-1,30$ & 26.80 & 12.1 & 17.3 & 70.6 & Silty clay \\
\hline $4-2,43$ & 28.43 & 14.7 & 31.0 & 54.4 & Silty clay \\
\hline $4-3,37$ & 29.87 & 19.1 & 24.9 & 56.0 & Silty clay \\
\hline $4-4,40$ & 31.40 & 16.6 & 29.7 & 53.7 & Silty clay \\
\hline $5-1,60$ & 36.60 & 25.7 & 26.9 & 47.3 & Sand-silt-clay \\
\hline $5-2,56$ & 38.06 & 13.1 & 18.6 & 68.3 & Silty clay \\
\hline $5-3,60$ & 39.60 & 16.7 & 23.1 & 60.2 & Silty clay \\
\hline $5-4,70$ & 41.20 & 16.7 & 20.9 & 62.4 & Silty clay \\
\hline $5-5,30$ & 42.30 & 15.4 & 33.3 & 51.2 & Silty clay \\
\hline $5-6,80$ & 44.30 & 8.8 & 40.3 & 50.8 & Silty clay \\
\hline $6-1,80$ & 46.30 & 11.7 & 26.2 & 52.1 & Silty clay \\
\hline $6-2,30$ & 47.30 & 9.0 & 26.0 & 65.0 & Silty clay \\
\hline $6-3,41$ & 48.91 & 14.8 & 27.4 & 57.8 & Silty clay \\
\hline $7-1,30$ & 55.30 & 11.8 & 24.4 & 63.8 & Silty clay \\
\hline $7-2,10$ & 56.60 & 12.1 & 29.2 & 58.6 & Silty clay \\
\hline $8-1,40$ & 64.90 & 10.5 & 32.5 & 57.0 & Silty clay \\
\hline $8-2,40$ & 66.40 & 11.4 & 24.5 & 64.1 & Silty clay \\
\hline $8-3,40$ & 67.90 & 11.4 & 27.0 & 61.6 & Silty clay \\
\hline $8-4,70$ & 69.70 & 10.7 & 28.8 & 60.6 & Silty clay \\
\hline $8-5,40$ & 70.90 & 13.1 & 25.5 & 61.5 & Silty clay \\
\hline $8-6,58$ & 72.58 & 7.4 & 31.6 & 61.0 & Silty clay \\
\hline $10-1,56$ & 84.06 & 12.9 & 27.2 & 59.9 & Silty clay \\
\hline $10-2,56$ & 85.56 & 13.4 & 28.5 & 58.1 & Silty clay \\
\hline $10-3,56$ & 87.06 & 13.7 & 25.0 & 61.3 & Silty clay \\
\hline $10-4,56$ & 88.56 & 8.0 & 28.9 & 63.1 & Silty clay \\
\hline $10-5,56$ & 90.06 & 9.1 & 29.7 & 61.2 & Silty clay \\
\hline $10-6,56$ & 91.56 & 11.2 & 26.4 & 62.4 & Silty clay \\
\hline $10-7,26$ & 92.76 & 11.9 & 24.1 & 64.0 & Silty clay \\
\hline $11-1,56$ & 93.56 & 11.5 & 22.9 & 65.5 & Silty clay \\
\hline $11-2,54$ & 95.04 & 10.9 & 26.1 & 63.0 & Silty clay \\
\hline $11-3,56$ & 96.56 & 14.8 & 26.1 & 59.1 & Silty clay \\
\hline $11-4,56$ & 98.06 & 13.0 & 25.5 & 61.4 & Silty clay \\
\hline $11-5,56$ & 99.56 & 10.9 & 27.2 & 61.9 & Silty clay \\
\hline $11-6,56$ & 101.06 & 7.9 & 35.7 & 56.4 & Silty clay \\
\hline $11-7,26$ & 102.26 & 8.5 & 22.9 & 68.5 & Silty clay \\
\hline $13-1,56$ & 112.56 & 8.0 & 27.2 & 64.8 & Silty clay \\
\hline $13-2,56$ & 114.06 & 10.1 & 28.1 & 61.8 & Silty clay \\
\hline $14-1,56$ & 122.06 & 7.7 & 31.6 & 60.7 & Silty clay \\
\hline $14-2,56$ & 123.56 & 7.3 & 30.6 & 62.1 & Silty clay \\
\hline $14-3,56$ & 125.06 & 5.8 & 30.1 & 64.1 & Silty clay \\
\hline $14-4,56$ & 126.56 & 11.2 & 27.5 & 61.3 & Silty clay \\
\hline $14-5,56$ & 128.06 & 6.3 & 26.6 & 67.2 & Silty clay \\
\hline $14-6,56$ & 129.56 & 8.6 & 28.1 & 63.3 & Silty clay \\
\hline $14-7,26$ & 130.76 & 8.0 & 31.6 & 60.4 & Silty clay \\
\hline $15-1,56$ & 131.56 & 7.6 & 27.6 & 64.9 & Silty clay \\
\hline $15-2,86$ & 133.36 & 8.6 & 27.5 & 63.9 & Silty clay \\
\hline $15-3,86$ & 134.86 & 8.0 & 27.8 & 64.2 & Silty clay \\
\hline $15-4,86$ & 136.36 & 10.5 & 25.3 & 64.2 & Silty clay \\
\hline $15-5,56$ & 137.56 & 11.3 & 23.0 & 65.7 & Silty clay \\
\hline
\end{tabular}

TABLE 1 - Continued

\begin{tabular}{|c|c|c|c|c|c|}
\hline $\begin{array}{c}\text { Sample } \\
\text { (Interval in cm) }\end{array}$ & $\begin{array}{l}\text { Sub-Bottom } \\
\text { Depth }\end{array}$ & $\begin{array}{l}\text { Sand } \\
(\%)\end{array}$ & $\begin{array}{l}\text { Silt } \\
(\%)\end{array}$ & $\begin{array}{r}\text { Clay } \\
(\%)\end{array}$ & Classification \\
\hline $15-6,56$ & 139.06 & 7.3 & 28.9 & 63.7 & Silty clay \\
\hline $16-1,56$ & 141.06 & 8.5 & 25.5 & 66.1 & Silty clay \\
\hline $16-2,56$ & 142.56 & 12.0 & 26.5 & 61.5 & Silty clay \\
\hline $16-3,56$ & 144.06 & 9.4 & 27.3 & 63.2 & Silty clay \\
\hline $16-4,56$ & 145.56 & 6.8 & 28.3 & 64.9 & Silty clay \\
\hline $16-5,56$ & 147.06 & 7.2 & 29.3 & 63.6 & Silty clay \\
\hline $16-6,56$ & 148.56 & 8.9 & 28.1 & 63.0 & Silty clay \\
\hline $18-1,80$ & 160.30 & 6.5 & 34.2 & 59.4 & Silty clay \\
\hline $18-2,80$ & 161.80 & 7.6 & 37.0 & 55.5 & Silty clay \\
\hline $18-3,70$ & 163.20 & 4.4 & 36.8 & 58.8 & Silty clay \\
\hline $18-4,50$ & 164.50 & 5.0 & 32.3 & 62.8 & Silty clay \\
\hline $18-5,40$ & 165.90 & 5.2 & 29.6 & 65.3 & Silty clay \\
\hline $19-1,92$ & 169.92 & 4.5 & 31.7 & 63.8 & Silty clay \\
\hline $19-2,50$ & 171.00 & 5.1 & 36.3 & 58.7 & Silty clay \\
\hline $19-3,40$ & 172.40 & 5.1 & 28.3 & 66.6 & Silty clay \\
\hline $19-4,90$ & 174.40 & 6.9 & 38.4 & 54.7 & Silty clay \\
\hline $19-5,58$ & 175.58 & 5.1 & 32.9 & 62.1 & Silty clay \\
\hline $19-6,20$ & 176.70 & 4.9 & 42.2 & 53.0 & Silty clay \\
\hline $20-1,90$ & 179.40 & 5.6 & 42.0 & 52.4 & Silty clay \\
\hline $20-2,69$ & 180.69 & 5.2 & 40.1 & 54.7 & Silty clay \\
\hline $20-3,33$ & 181.83 & 5.0 & 40.2 & 54.8 & Silty clay \\
\hline $21-1,98$ & 188.98 & 3.8 & 38.5 & 57.7 & Silty clay \\
\hline $21-2,70$ & 190.20 & 3.2 & 36.1 & 60.8 & Silty clay \\
\hline $21-3,60$ & 191.60 & 3.9 & 33.2 & 62.8 & Silty clay \\
\hline $21-4,40$ & 192.90 & 2.0 & 38.3 & 59.7 & Silty clay \\
\hline $21-5,88$ & 194.88 & 2.0 & 36.3 & 61.7 & Silty clay \\
\hline $21-6,31$ & 195.81 & 2.3 & 36.4 & 61.3 & Silty clay \\
\hline $21-7,10$ & 197.10 & 2.7 & 37.8 & 59.5 & Silty clay \\
\hline $22-1,100$ & 198.50 & 2.3 & 39.9 & 57.8 & Silty clay \\
\hline $22-2,90$ & 199.90 & 2.1 & 38.1 & 59.8 & Silty clay \\
\hline $22-3,88$ & 201.38 & 2.3 & 38.3 & 59.4 & Silty clay \\
\hline $22-4,50$ & 202.50 & 2.6 & 39.2 & 58.2 & Silty clay \\
\hline $22-5,70$ & 204.20 & 2.0 & 92.8 & 5.3 & Silt \\
\hline $22-6,70$ & 205.70 & 2.4 & 40.2 & 57.5 & Silty clay \\
\hline $22-7,24$ & 206.74 & 2.4 & 35.7 & 61.9 & Silty clay \\
\hline $23-1,74$ & 207.74 & 1.9 & 39.0 & 59.1 & Silty clay \\
\hline $23-2,100$ & 209.50 & 2.0 & 38.5 & 59.5 & Silty clay \\
\hline $23-3,80$ & 210.80 & 1.5 & 39.3 & 59.3 & Silty clay \\
\hline $23-4,90$ & 212.40 & 1.1 & 37.9 & 61.0 & Silty clay \\
\hline $23-5,70$ & 213.70 & 1.9 & 41.6 & 56.5 & Silty clay \\
\hline $23-6,40$ & 214.90 & 1.7 & 32.1 & 66.2 & Silty clay \\
\hline $24-1,68$ & 217.18 & 2.7 & 31.0 & 66.4 & Silty clay \\
\hline $24-2,87$ & 218.87 & 2.2 & 33.8 & 63.9 & Silty clay \\
\hline $24-3,68$ & 220.18 & 2.0 & 37.9 & 60.1 & Silty clay \\
\hline $24-4,46$ & 221.46 & 2.2 & 34.1 & 63.7 & Silty clay \\
\hline $24-5,78$ & 223.28 & 2.7 & 31.6 & 65.8 & Silty clay \\
\hline $24-6,18$ & 224.18 & 2.4 & 31.7 & 65.9 & Silty clay \\
\hline $24-7,18$ & 225.68 & 1.2 & 32.2 & 66.6 & Silty clay \\
\hline $25-1,78$ & 226.78 & 2.4 & 34.6 & 63.0 & Silty clay \\
\hline $25-2,56$ & 228.06 & 2.3 & 34.3 & 63.4 & Silty clay \\
\hline $25-3,68$ & 229.68 & 3.8 & 35.6 & 60.6 & Silty clay \\
\hline $25-4,56$ & 231.06 & 2.4 & 33.0 & 64.5 & Silty clay \\
\hline $25-5,56$ & 232.56 & 3.3 & 35.6 & 61.1 & Silty clay \\
\hline $25-6,56$ & 234.06 & 2.4 & 33.0 & 64.6 & Silty clay \\
\hline $25-7,26$ & 235.26 & 2.0 & 41.6 & 56.4 & Silty clay \\
\hline $26-1,36$ & 235.86 & 4.1 & 41.2 & 54.7 & Silty clay \\
\hline $26-2,36$ & 237.36 & 2.1 & 40.6 & 57.3 & Silty clay \\
\hline $26-3,36$ & 238.86 & 3.5 & 41.8 & 54.7 & Silty clay \\
\hline $27-1,56$ & 245.56 & 8.2 & 39.1 & 52.7 & Silty clay \\
\hline $27-2,56$ & 247.06 & 4.8 & 36.5 & 58.7 & Silty clay \\
\hline $27-3,56$ & 248.56 & 4.3 & 38.8 & 56.9 & Silty clay \\
\hline $28-1,56$ & 255.06 & 9.1 & 37.7 & 53.2 & Silty clay \\
\hline $28-2,56$ & 256.56 & 7.6 & 43.6 & 48.9 & Silty clay \\
\hline $28-3,56$ & 258.06 & 5.6 & 40.2 & 54.3 & Silty clay \\
\hline $28-4,56$ & 259.56 & 7.4 & 33.3 & 59.3 & Silty clay \\
\hline $29-1,56$ & 264.56 & 5.9 & 31.7 & 62.4 & Silty clay \\
\hline $29-2,45$ & 265.95 & 10.3 & 33.1 & 56.6 & Silty clay \\
\hline $29-3,56$ & 267.56 & 6.1 & 36.1 & 57.8 & Silty clay \\
\hline
\end{tabular}


TABLE 1 - Continued

\begin{tabular}{|c|c|c|c|c|c|}
\hline $\begin{array}{c}\text { Sample } \\
\text { (Interval in } \mathrm{cm} \text { ) }\end{array}$ & $\begin{array}{l}\text { Sub-Bottom } \\
\text { Depth }\end{array}$ & $\begin{array}{l}\text { Sand } \\
(\%)\end{array}$ & $\begin{array}{l}\text { Silt } \\
(\%)\end{array}$ & $\begin{array}{r}\text { Clay } \\
(\%)\end{array}$ & Classification \\
\hline $29-4,56$ & 269.06 & 6.7 & 33.7 & 59.6 & Silty clay \\
\hline $29-5,26$ & 270.26 & 8.2 & 39.0 & 52.7 & Silty clay \\
\hline $30-1,56$ & 274.06 & 8.3 & 31.1 & 60.7 & Silty clay \\
\hline $30-2,56$ & 275.56 & 5.6 & 36.8 & 57.6 & Silty clay \\
\hline $30-3,56$ & 277.06 & 7.5 & 37.0 & 55.6 & Silty clay \\
\hline $30-4,56$ & 278.56 & 5.3 & 36.9 & 57.7 & Silty clay \\
\hline $30-5,56$ & 280.06 & 7.4 & 40.8 & 51.7 & Silty clay \\
\hline $30-6,56$ & 281.56 & 4.4 & 42.8 & 52.7 & Silty clay \\
\hline $30-7,26$ & 282.76 & 4.4 & 41.3 & 54.3 & Silty clay \\
\hline $31-1,83$ & 283.83 & 4.1 & 42.5 & 53.4 & Silty clay \\
\hline $31-2,77$ & 285.27 & 4.8 & 42.4 & 52.8 & Silty clay \\
\hline $31-3,60$ & 286.60 & 3.7 & 46.7 & 49.6 & Silty clay \\
\hline $31-4,33$ & 287.83 & 3.9 & 45.8 & 50.2 & Silty clay \\
\hline $32-1,61$ & 293.11 & 2.6 & 35.0 & 62.3 & Silty clay \\
\hline $32-2,50$ & 294.50 & 3.0 & 42.5 & 54.5 & Silty clay \\
\hline $32-3,79$ & 296.29 & 2.1 & 42.1 & 55.8 & Silty clay \\
\hline $33-1,57$ & 302.57 & 3.7 & 46.4 & 49.9 & Silty clay \\
\hline $33-2,45$ & 303.95 & 3.5 & 50.6 & 45.9 & Clayey silt \\
\hline $34-1,15$ & 311.65 & 26. & 11.7 & 85.7 & Clay \\
\hline $34-2,80$ & 313.80 & 2.9 & 45.8 & 51.3 & Silty clay \\
\hline $35-1,76$ & 321.76 & 3.0 & 50.2 & 46.8 & Clayey silt \\
\hline $35-2,75$ & 323.25 & 1.8 & 49.8 & 48.4 & Clayey silt \\
\hline $36-1,70$ & 331.20 & 2.6 & 48.2 & 49.2 & Silty clay \\
\hline $36-2,136$ & 333.36 & 3.5 & 48.4 & 48.2 & Clayey silt \\
\hline $36-3,49$ & 333.99 & 1.1 & 50.6 & 48.2 & Clayey silt \\
\hline \multicolumn{6}{|l|}{ Hole 410A } \\
\hline $1-1,70$ & 325.70 & 25.7 & 40.6 & 33.7 & Sand-silt-clay \\
\hline $1-2,90$ & 327.40 & 18.7 & 22.5 & 58.8 & Silty clay \\
\hline $1-3,60$ & 328.60 & 22.4 & 22.9 & 54.7 & Sand-silt-clay \\
\hline $1-4,70$ & 330.20 & 18.3 & 22.0 & 59.6 & Silty clay \\
\hline $1-5,87$ & 331.87 & 1.6 & 37.4 & 61.0 & Silty clay \\
\hline $1-6,19$ & 332.69 & 4.2 & 47.5 & 48.2 & Silty clay \\
\hline \multicolumn{6}{|l|}{ Hole 411} \\
\hline $1-1,0$ & 74.00 & 77.8 & 10.1 & 12.1 & Sand \\
\hline \multicolumn{6}{|l|}{ Hole 412} \\
\hline $1-1,53$ & 0.53 & 12.6 & 29.0 & 58.4 & Silty clay \\
\hline $1-1,107$ & 1.07 & 8.2 & 29.1 & 62.7 & Silty clay \\
\hline $1-2,56$ & 2.06 & 8.5 & 32.4 & 59.2 & Silty clay \\
\hline $2-1,56$ & 4.56 & 9.7 & 30.0 & 60.3 & Silty clay \\
\hline $2-2,56$ & 6.06 & 9.1 & 31.0 & 59.9 & Silty clay \\
\hline $2-3,56$ & 7.56 & 9.8 & 28.4 & 61.7 & Silty clay \\
\hline $2-4,56$ & 9.06 & 8.4 & 30.2 & 61.4 & Silty clay \\
\hline $2-5,56$ & 10.56 & 6.5 & 29.0 & 64.5 & Silty clay \\
\hline $2-6,66$ & 12.16 & 11.2 & 24.5 & 64.3 & Silty clay \\
\hline $3-1,56$ & 14.06 & 8.7 & 25.2 & 66.1 & Silty clay \\
\hline $3-2,56$ & 15.56 & 6.5 & 31.3 & 62.2 & Silty clay \\
\hline $3-3,56$ & 17.06 & 6.1 & 29.6 & 64.3 & Silty clay \\
\hline $3-4,56$ & 18.36 & 9.8 & 28.1 & 62.1 & Silty clay \\
\hline $3-5,66$ & 20.16 & 9.6 & 28.2 & 62.2 & Silty clay \\
\hline $3-6,56$ & 21.56 & 5.6 & 29.2 & 65.2 & Silty clay \\
\hline $4-1,56$ & 23.56 & 6.7 & 31.1 & 62.2 & Silty clay \\
\hline $4-2,56$ & 25.06 & 9.0 & 30.3 & 60.7 & Silty clay \\
\hline $4-3,56$ & 26.56 & 9.0 & 24.7 & 66.3 & Silty clay \\
\hline $4-4,56$ & 28.06 & 7.0 & 17.2 & 75.9 & Clay \\
\hline $4-5,56$ & 29.56 & 7.1 & 19.4 & 73.4 & Silty clay \\
\hline $4-6,56$ & 31.06 & 8.7 & 20.5 & 70.8 & Silty clay \\
\hline $5-1,56$ & 52.06 & 4.2 & 16.6 & 79.2 & Clay \\
\hline $5-2,56$ & 53.56 & 6.4 & 17.7 & 75.9 & Clay \\
\hline $5-3,56$ & 55.06 & 3.5 & 16.0 & 80.5 & Clay \\
\hline $5-4,56$ & 56.56 & 5.1 & 12.4 & 82.5 & Clay \\
\hline $6-1,56$ & 80.56 & 7.6 & 22.1 & 70.3 & Silty clay \\
\hline $6-2,56$ & 82.06 & 15.7 & 26.0 & 58.3 & Silty clay \\
\hline $6-3,56$ & 83.56 & 9.4 & 28.7 & 61.8 & Silty clay \\
\hline $6-4,36$ & 84.86 & 14.8 & 24.0 & 61.2 & Silty clay \\
\hline
\end{tabular}

TABLE 1 - Continued

\begin{tabular}{|c|c|c|c|c|c|}
\hline $\begin{array}{c}\text { Sample } \\
\text { (Interval in } \mathrm{cm} \text { ) }\end{array}$ & $\begin{array}{l}\text { Sub-Bottom } \\
\text { Depth }\end{array}$ & $\begin{array}{l}\text { Sand } \\
(\%)\end{array}$ & $\begin{array}{l}\text { Silt } \\
(\%)\end{array}$ & $\begin{array}{r}\text { Clay } \\
(\%)\end{array}$ & Classification \\
\hline $7-1,56$ & 90.06 & 8.1 & 27.0 & 64.9 & Silty clay \\
\hline $7-2,56$ & 91.56 & 9.7 & 26.7 & 63.6 & Silty clay \\
\hline $8-1,64$ & 99.64 & 7.0 & 28.8 & 64.2 & Silty clay \\
\hline $8-2,64$ & 101.14 & 3.8 & 29.8 & 66.3 & Silty clay \\
\hline $8-3,64$ & 102.64 & 4.6 & 29.4 & 66.0 & Silty clay \\
\hline $8-4,64$ & 104.14 & 9.3 & 28.2 & 62.5 & Silty clay \\
\hline $8-564$ & 105.64 & 9.8 & 24.9 & 65.4 & Silty clay \\
\hline $8-6,64$ & 107.14 & 9.1 & 27.0 & 63.8 & Silty clay \\
\hline $8-7,21$ & 108.21 & 7.4 & 29.6 & 63.0 & Silty clay \\
\hline $9-1,100$ & 109.50 & 10.5 & 25.4 & 64.0 & Silty clay \\
\hline $9-2,70$ & 110.70 & 9.3 & 27.2 & 63.5 & Silty clay \\
\hline $9-3,70$ & 112.20 & 8.3 & 25.9 & 65.9 & Silty clay \\
\hline $9-4,60$ & 113.60 & 5.6 & 26.1 & 68.3 & Silty clay \\
\hline $9-5,0$ & 114.50 & 6.2 & 22.6 & 71.2 & Silty clay \\
\hline $9-6,50$ & 116.50 & 7.4 & 27.3 & 65.3 & Silty clay \\
\hline $10-1,60$ & 118.60 & 6.2 & 26.4 & 67.5 & Silty clay \\
\hline $10-2,60$ & 120.10 & 8.5 & 28.0 & 63.5 & Silty clay \\
\hline $10-3,100$ & 122.00 & 9.9 & 28.2 & 61.9 & Silty clay \\
\hline $10-4,60$ & 123.10 & 9.0 & 25.6 & 65.4 & Silty clay \\
\hline $10-5,10$ & 124.10 & 9.2 & 28.3 & 62.6 & Silty clay \\
\hline $10-6,10$ & 125.60 & 8.9 & 26.5 & 64.6 & Silty clay \\
\hline $12-1,60$ & 137.60 & 7.2 & 22.2 & 70.6 & Silty clay \\
\hline $12-2,60$ & 139.10 & 12.0 & 27.4 & 60.5 & Silty clay \\
\hline $12-3,10$ & 140.10 & 11.6 & 25.9 & 62.5 & Silty clay \\
\hline $13-1,7$ & 146.57 & 15.2 & 25.6 & 59.2 & Silty clay \\
\hline $14-1,58$ & 156.58 & 5.5 & 30.7 & 63.8 & Silty clay \\
\hline $14-2,60$ & 158.10 & 6.3 & 30.7 & 63.0 & Silty clay \\
\hline $14-3,60$ & 159.60 & 6.0 & 33.8 & 60.2 & Silty clay \\
\hline $14-4,50$ & 161.00 & 3.7 & 34.3 & 62.1 & Silty clay \\
\hline $14-5,60$ & 162.60 & 3.7 & 32.8 & 63.5 & Silty clay \\
\hline $14-6,60$ & 164.10 & 2.6 & 32.9 & 64.6 & Silty clay \\
\hline $14-7,10$ & 165.10 & 17.1 & 28.7 & 54.2 & Silty clay \\
\hline
\end{tabular}

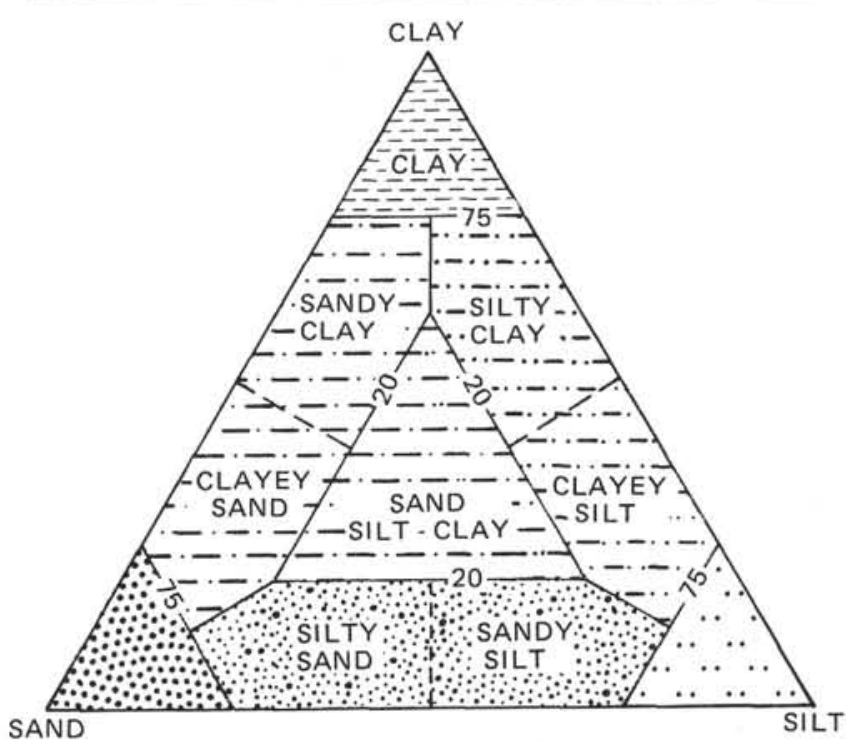

Figure 1. Sediment classification after Shepard (1954) with the sand, silt, and clay size fractions based on the Wentworth (1922) Grade Scale: Sand, silt, and clay size particles having respective diameters of 2000 to $62.5 \mu \mathrm{m}$, 62.5 to $3.91 \mu \mathrm{m}$, and less than $3.91 \mu \mathrm{m}$. Shepard's (1954) sediment classification is a function of sand, silt, and clay size percentages and not composition. 
TABLE 2

Carbon and Carbonate Analyses, Leg 49

\begin{tabular}{|c|c|c|c|c|c|c|c|c|c|}
\hline $\begin{array}{c}\text { Sample } \\
\text { (Interval in cm) }\end{array}$ & $\begin{array}{l}\text { Depth } \\
\text { (m) }\end{array}$ & $\begin{array}{c}\text { Total } \\
\text { Carbon (\%) }\end{array}$ & $\begin{array}{c}\text { Organic } \\
\text { Carbon (\%) }\end{array}$ & $\mathrm{CaCO}_{3}$ & $\begin{array}{c}\text { Sample } \\
\text { (Interval in } \mathrm{cm} \text { ) }\end{array}$ & $\begin{array}{c}\text { Depth } \\
\text { (m) }\end{array}$ & $\begin{array}{l}\text { Total } \\
\text { Carbon (\%) }\end{array}$ & $\begin{array}{c}\text { Organic } \\
\text { Carbon (\%) }\end{array}$ & $\mathrm{CaCO}_{3}$ \\
\hline Hole 407 & & & & & $19-3,70$ & 171.20 & 7.3 & 0.1 & 60 \\
\hline $1-1,3$ & 0.03 & 7.4 & 0.1 & 61 & $19-4,70$ & 172.70 & 0.7 & 0.1 & 5 \\
\hline $1-1,26$ & 0.26 & 1.9 & 0.1 & 15 & $19-5,70$ & 174.20 & 72. & 0.1 & 59 \\
\hline $1-2,6$ & 1.56 & 4.2 & 0.1 & 34 & $20-1,41$ & 177.41 & 7.6 & 0.1 & 62 \\
\hline $1-2,87$ & 2.37 & 2.7 & 0.1 & 22 & $20-2,41$ & 178.91 & 9.2 & 0.1 & 76 \\
\hline $1-3,20$ & 3.20 & 2.2 & 0.1 & 18 & $21-1,41$ & 186.91 & 5.1 & 0.2 & 42 \\
\hline $1-4,12$ & 4.62 & 2.0 & 0.1 & 16 & $22-1,70$ & 196.70 & 6.3 & 0.1 & 52 \\
\hline $1-4,78$ & 5.28 & 1.4 & 0.2 & 10 & $22-2,70$ & 198.20 & 6.1 & 0.2 & 49 \\
\hline $2-1,33$ & 6.33 & 1.5 & 0.1 & 11 & $22-3,70$ & 199.70 & 5.7 & 0.2 & 46 \\
\hline $2-1,119$ & 7.19 & 6.7 & 0.1 & 56 & $23-1,70$ & 206.20 & 7.2 & 0.1 & 59 \\
\hline $2-3,41$ & 9.41 & 8.1 & 0.1 & 67 & $23-2,70$ & 207.70 & 7.5 & 0.1 & 61 \\
\hline $2-4,41$ & 10.91 & 2.0 & 0.1 & 16 & $23-3,70$ & 209.20 & 7.9 & 0.1 & 65 \\
\hline $2-5,85$ & 12.85 & 1.7 & 0.1 & 13 & $24-1,70$ & 215.70 & 2.4 & 0.2 & 18 \\
\hline $2-6,45$ & 13.95 & 2.6 & 0.1 & 20 & $24-2,70$ & 217.20 & 0.4 & 0.3 & 1 \\
\hline $3-1,42$ & 15.92 & 6.1 & 0.1 & 50 & $24-3,70$ & 218.70 & 8.7 & 0.1 & 72 \\
\hline $3-2,114$ & 18.14 & 2.4 & 0.1 & 19 & $24-4,70$ & 220.20 & 7.3 & 0.1 & 60 \\
\hline $3-4,28$ & 20.28 & 1.7 & 0.2 & 12 & $24-5,70$ & 221.70 & 7.7 & 0.1 & 63 \\
\hline $4-1,92$ & 25.92 & 1.9 & 0.1 & 15 & $24-6,70$ & 223.20 & 5.8 & 0.1 & 47 \\
\hline $4-2,34$ & 26.84 & 1.0 & 0.2 & 7 & $24-7,8$ & 224.08 & 7.2 & 0.1 & 59 \\
\hline $4-3,98$ & 28.98 & 6.9 & 0.1 & 57 & $26-1,38$ & 234.38 & 9.3 & 0.1 & 77 \\
\hline $4-4,82$ & 30.32 & 0.9 & 0.1 & 6 & $28-1,68$ & 253.68 & 6.1 & 0.2 & 49 \\
\hline $5-1,81$ & 35.31 & 2.3 & 0.1 & 18 & $28-2,68$ & 255.18 & 6.5 & 0.1 & 53 \\
\hline $5-2,33$ & 36.33 & 1.0 & 0.1 & 8 & $28-3,68$ & 256.68 & 4.1 & 0.1 & 33 \\
\hline $5-3,8$ & 37.58 & 1.5 & 0.1 & 12 & $28-4,68$ & 258.18 & 4.9 & 0.1 & 40 \\
\hline $6-1,23$ & 44.23 & 1.2 & 0.1 & 9 & $28-5,70$ & 259.70 & 5.4 & 0.1 & 44 \\
\hline $6-2,59$ & 46.09 & 6.2 & 0.1 & 51 & $29-1,55$ & 263.05 & 0.4 & 0.1 & 3 \\
\hline $6-3,62$ & 47.62 & 8.1 & 0.1 & 67 & $29-5,20$ & 268.70 & 5.3 & 0.1 & 43 \\
\hline $7-1,75$ & 54.25 & 8.3 & 0.1 & 69 & $30-1,66$ & 272.66 & 5.7 & 0.1 & 47 \\
\hline $7-2,69$ & 55.69 & 8.1 & 0.1 & 67 & $30-2,65$ & 274.15 & 5.4 & 0.2 & 44 \\
\hline $8-1,30$ & 63.30 & 7.6 & 0.1 & 62 & $30-3,63$ & 275.63 & 5.8 & 0.2 & 47 \\
\hline $8-2,30$ & 64.80 & 7.5 & 0.1 & 62 & $30-4,67$ & 277.17 & 6.2 & 0.2 & 50 \\
\hline $8-3,10$ & 66.10 & 8.9 & 0.1 & 74 & $30-5,67$ & 278.67 & 5.8 & & \\
\hline $9-2,70$ & 74.70 & 8.5 & 0.1 & 70 & $30-6,33$ & 279.83 & 5.1 & 0.2 & 41 \\
\hline $10-1,10$ & 82.10 & 9.1 & 0.1 & 75 & $31-1,70$ & 282.20 & 6.3 & 0.2 & 51 \\
\hline $10-2,10$ & 83.60 & 9.5 & 0.1 & 79 & $31-2,70$ & 283.70 & 6.5 & 0.2 & 53 \\
\hline $10-3,92$ & 85.92 & 9.7 & 0.1 & 80 & $31-3,68$ & 285.18 & 6.1 & 0.2 & 49 \\
\hline $10-4,20$ & 96.70 & 9.5 & 0.1 & 79 & $31-4,70$ & 286.70 & 6.7 & 0.2 & 54 \\
\hline $11-1,71$ & 92.21 & 9.4 & 0.1 & 78 & $31-5,59$ & 288.09 & 6.0 & 0.2 & 49 \\
\hline $11-2,71$ & 93.71 & 9.6 & 0.1 & 80 & $31-6,70$ & 289.70 & 4.5 & 0.2 & 36 \\
\hline $11-3,71$ & 95.21 & 9.0 & 0.1 & 74 & $31-7,23$ & 290.73 & 4.2 & 0.1 & 34 \\
\hline $11-4,71$ & 96.71 & 10.2 & 0.1 & 84 & $32-1,26$ & 291.26 & & 0.1 & \\
\hline $12-1,70$ & 101.70 & 10.1 & 0.1 & 83 & & & & & \\
\hline $12-2,70$ & 103.20 & 10.5 & 0.1 & 87 & Hole 408 & & & & \\
\hline $12-3,70$ & 104.70 & 9.8 & 0.1 & 81 & $1-1,40$ & 0.40 & 3.0 & 0.1 & 24 \\
\hline $13-1,70$ & 111.20 & 9.7 & 0.1 & 80 & $1-2,31$ & 1.81 & 8.0 & 0.1 & 66 \\
\hline $13-2,79$ & 112.79 & 9.3 & 0.1 & 77 & $1-3,14$ & 3.14 & 5.7 & 0.1 & 47 \\
\hline $13-3,79$ & 114.29 & 9.8 & 0.1 & 81 & $1-4,23$ & 4.73 & 1.8 & 0.2 & 14 \\
\hline $13-4,79$ & 115.79 & 9.4 & 0.1 & 78 & $1-5,60$ & 6.60 & 3.7 & 0.1 & 30 \\
\hline $13-5,50$ & 117.00 & 9.3 & 0.1 & 77 & $1-6,40$ & 7.90 & 2.5 & 0.1 & 20 \\
\hline $13-6,50$ & 118.50 & 8.7 & 0.1 & 72 & $2-1,43$ & 9.93 & 1.4 & 0.1 & 11 \\
\hline $14-1,70$ & 120.70 & 8.4 & 0.1 & 69 & $2-2,30$ & 11.30 & 2.4 & 0.1 & 19 \\
\hline $14-2,70$ & 122.20 & 9.6 & 0.1 & 79 & $2-3,20$ & 12.70 & 7.0 & 0.1 & 57 \\
\hline $14-3,70$ & 123.70 & 8.4 & 0.1 & 69 & $2-4,50$ & 14.50 & 1.9 & 0.2 & 14 \\
\hline $14-4,25$ & 124.75 & 0.9 & 0.1 & 7 & $2-5,50$ & 16.00 & 0.4 & 0.1 & 3 \\
\hline $16-1,87$ & 139.87 & 8.0 & 0.1 & 66 & $3-1,49$ & 19.49 & 0.6 & 0.1 & 4 \\
\hline $16-2,87$ & 141.37 & 9.9 & 0.1 & 82 & $3-2,50$ & 21.00 & 1.1 & 0.1 & 8 \\
\hline $16-3,87$ & 142.87 & 9.2 & 0.1 & 76 & $3-3,55$ & 22.55 & 3.1 & 0.1 & 25 \\
\hline $16-4,3$ & 143.53 & 9.4 & 0.1 & 78 & 34,50 & 24.00 & 2.8 & 0.1 & 23 \\
\hline $17-1,70$ & 149.20 & 10.0 & 0.1 & 83 & $3-5,14$ & 25.14 & 0.4 & 0.1 & 3 \\
\hline $17-2,70$ & 150.70 & 8.5 & 0.1 & 70 & $4-1,41$ & 28.91 & 0.3 & 0.1 & 1 \\
\hline $17-3,70$ & 152.20 & 9.8 & 0.1 & 81 & $4-2,50$ & 30.50 & 1.7 & 0.1 & 14 \\
\hline $18-1,70$ & 158.70 & 9.0 & 0.1 & 74 & $4-3,50$ & 32.00 & 2.8 & 0.1 & 22 \\
\hline $18-2,70$ & 160.20 & 4.0 & 0.1 & 32 & $4-4,50$ & 33.50 & 1.3 & 0.1 & 10 \\
\hline $18-3,70$ & 161.70 & 7.4 & 0.1 & 61 & $4-5,50$ & 35.00 & 0.5 & 0.2 & 3 \\
\hline $18-4,70$ & 163.20 & 7.6 & 0.1 & 63 & $5-1,64$ & 38.64 & 2.8 & 0.2 & 22 \\
\hline $18-5,70$ & 164.70 & 7.4 & 0.1 & 61 & $5-2,70$ & 40.20 & 3.3 & 0.1 & 27 \\
\hline $19-1,70$ & 168.20 & 7.7 & 0.1 & 63 & $5-3,70$ & 41.70 & 3.9 & 0.1 & 31 \\
\hline $19-2,70$ & 169.70 & 7.5 & 0.1 & 62 & $5-4,40$ & 42.90 & 5.5 & 0.1 & 45 \\
\hline
\end{tabular}


TABLE 2 - Continued

\begin{tabular}{|c|c|c|c|c|}
\hline $\begin{array}{c}\text { Sample } \\
\text { (Interval in } \mathrm{cm} \text { ) }\end{array}$ & $\begin{array}{c}\text { Depth } \\
\text { (m) }\end{array}$ & $\begin{array}{l}\text { Total } \\
\text { Carbon (\%) }\end{array}$ & $\begin{array}{c}\text { Organic } \\
\text { Carbon (\%) }\end{array}$ & $\mathrm{CaCO}_{3}$ \\
\hline $6-1,50$ & 48.00 & 7.9 & 0.1 & 65 \\
\hline $6-2,80$ & 49.80 & 9.4 & 0.1 & 77 \\
\hline $6-3,50$ & 51.00 & 5.3 & 0.1 & 44 \\
\hline $7-1,50$ & 57.50 & 9.8 & 0.1 & 81 \\
\hline $7-2,20$ & 58.70 & 10.1 & 0.1 & 84 \\
\hline $8-1,40$ & 66.90 & 9.1 & 0.1 & 75 \\
\hline $8-2,52$ & 68.52 & 9.5 & 0.1 & 78 \\
\hline $9-1,40$ & 76.40 & 10.2 & 0.1 & 84 \\
\hline $9-2,40$ & 77.90 & 10.7 & 0.1 & 89 \\
\hline $10-1,50$ & 86.00 & 9.7 & 0.1 & 81 \\
\hline $10-2,50$ & 87.50 & 9.9 & 0.1 & 82 \\
\hline $10-3,50$ & 89.00 & 10.3 & 0.1 & 85 \\
\hline $10-4,50$ & 90.50 & 10.0 & 0.1 & 83 \\
\hline $10-5,20$ & 91.70 & 10.4 & 0.1 & 86 \\
\hline $11-1,50$ & 95.50 & 9.7 & 0.1 & 80 \\
\hline $11-2,50$ & 97.00 & 10.5 & 0.1 & 87 \\
\hline $11-3,50$ & 98.50 & 9.9 & 0.1 & 82 \\
\hline $11-4,50$ & 100.00 & 7.7 & 0.1 & 63 \\
\hline $11-5,50$ & 101.50 & 9.7 & 0.1 & 80 \\
\hline $11-6,30$ & 102.80 & 9.5 & 0.1 & 79 \\
\hline $12-1,50$ & 105.00 & 9.5 & 0.1 & 78 \\
\hline $12-2,50$ & 106.50 & 8.9 & 0.1 & 73 \\
\hline $13-1,50$ & 114.50 & 9.2 & 0.1 & 76 \\
\hline $13-2,50$ & 116.00 & 10.6 & 0.1 & 88 \\
\hline $13-3,50$ & 117.50 & 10.6 & 0.1 & 87 \\
\hline $15-1,50$ & 133.50 & 10.2 & 0.1 & 84 \\
\hline $15-2,90$ & 135.40 & 10.3 & 0.1 & 85 \\
\hline $15-3,50$ & 136.50 & 9.6 & 0.1 & 79 \\
\hline $15-4,50$ & 138.00 & 10.2 & 0.1 & 85 \\
\hline $15-5,50$ & 139.50 & 11.0 & 0.1 & 81 \\
\hline $16-1,100$ & 143.50 & 11.0 & 0.1 & 81 \\
\hline $16-2,50$ & 144.50 & 11.1 & 0.1 & 82 \\
\hline $16-3,20$ & 145.70 & 11.2 & 0.1 & 83 \\
\hline $17-1,50$ & 152.50 & 10.4 & 0.1 & 86 \\
\hline $17-2,50$ & 154.00 & 10.1 & 0.1 & 84 \\
\hline $17-3,50$ & 155.50 & 10.7 & 0.1 & 89 \\
\hline $17-4,50$ & 157.00 & 10.6 & 0.1 & 88 \\
\hline $17-5,50$ & 158.50 & 10.4 & 0.1 & 86 \\
\hline $18-1,40$ & 161.90 & 10.1 & 0.1 & 83 \\
\hline $18-2,20$ & 163.20 & 10.2 & 0.1 & 84 \\
\hline $18-3,50$ & 165.00 & 9.5 & 0.1 & 79 \\
\hline $18-4,10$ & 166.10 & 9.9 & 0.1 & 81 \\
\hline $19-1,20$ & 171.20 & 11.1 & 0.1 & 92 \\
\hline $19-2,50$ & 173.00 & 11.0 & 0.1 & 91 \\
\hline $19-3,50$ & 174.50 & 11.0 & 0.1 & 91 \\
\hline $19-4,50$ & 176.00 & 10.8 & 0.1 & 89 \\
\hline $19-5,50$ & 177.50 & 10.6 & 0.1 & 87 \\
\hline $19-6,50$ & 179.00 & 10.1 & 0.1 & 84 \\
\hline $19-7,10$ & 180.10 & 10.9 & 0.1 & 90 \\
\hline $20-1,50$ & 181.00 & 10.9 & 0.1 & 90 \\
\hline $20-2,50$ & 182.50 & 10.4 & 0.1 & 86 \\
\hline $21-1,50$ & 190.50 & 9.3 & 0.1 & 77 \\
\hline $21-2,50$ & 192.00 & 9.3 & 0.1 & 77 \\
\hline $21-3,50$ & 193.50 & 9.7 & 0.1 & 80 \\
\hline $21-4,50$ & 195.00 & 8.7 & 0.1 & 71 \\
\hline $22-1,50$ & 200.00 & 7.9 & 0.1 & 65 \\
\hline $22-2,50$ & 201.50 & 10.1 & 0.1 & 84 \\
\hline $22-3,50$ & 203.00 & 10.1 & 0.1 & 84 \\
\hline $23-1,73$ & 209.73 & 8.8 & 0.1 & 73 \\
\hline $23-2,70$ & 211.20 & 8.6 & 0.1 & 71 \\
\hline $23-3,33$ & 212.33 & 10.1 & 0.1 & 83 \\
\hline $24-1,50$ & 219.00 & 7.0 & 0.1 & 57 \\
\hline $24-2,60$ & 220.60 & 5.0 & 0.1 & 41 \\
\hline $24-3,47$ & 221.97 & 5.4 & 0.1 & 44 \\
\hline $24-5,40$ & 224.90 & 8.3 & 0.1 & 69 \\
\hline $24-6,35$ & 226.35 & 8.6 & 0.1 & 71 \\
\hline $25-1,49$ & 228.49 & 9.5 & 0.1 & 78 \\
\hline $25-2,46$ & 229.96 & 9.3 & 0.1 & 76 \\
\hline
\end{tabular}

TABLE 2 - Continued

\begin{tabular}{|c|c|c|c|c|}
\hline $\begin{array}{c}\text { Sample } \\
\text { (Interval in } \mathrm{cm} \text { ) }\end{array}$ & $\begin{array}{c}\text { Depth } \\
\text { (m) }\end{array}$ & $\begin{array}{l}\text { Total } \\
\text { Carbon (\%) }\end{array}$ & $\begin{array}{c}\text { Organic } \\
\text { Carbon (\%) }\end{array}$ & $\mathrm{CaCO}_{3}$ \\
\hline $25-3,59$ & 231.59 & 8.4 & 0.1 & 69 \\
\hline $25-4,60$ & 233.10 & 8.4 & 0.1 & 70 \\
\hline $25-5,67$ & 234.67 & 8.7 & 0.1 & 72 \\
\hline $25-6,66$ & 236.16 & 8.3 & 0.1 & 69 \\
\hline $26-1,66$ & 238.16 & 8.6 & 0.1 & 71 \\
\hline $26-2,60$ & 239.60 & 7.8 & 0.1 & 64 \\
\hline $26-3,50$ & 241.00 & 8.2 & 0.1 & 67 \\
\hline $26-4,50$ & 242.50 & 7.5 & 0.1 & 62 \\
\hline $26-5,60$ & 244.10 & 7.2 & 0.1 & 59 \\
\hline $27-1,60$ & 247.60 & 6.2 & 0.1 & 51 \\
\hline $27-2,60$ & 249.10 & 8.1 & 0.1 & 66 \\
\hline $27-3,60$ & 250.60 & 7.8 & 0.1 & 64 \\
\hline $27-4,66$ & 252.16 & 6.0 & 0.1 & 49 \\
\hline $27-5,60$ & 253.60 & 5.7 & 0.1 & 46 \\
\hline $27-6,60$ & 255.10 & 7.9 & 0.1 & 65 \\
\hline $28-1,60$ & 257.10 & 3.1 & 0.1 & 25 \\
\hline $28-2,60$ & 258.60 & 6.7 & 0.1 & 55 \\
\hline $28-3,60$ & 260.10 & 7.5 & 0.1 & 61 \\
\hline $29-1,60$ & 266.60 & 7.7 & 0.2 & 63 \\
\hline $29-2,60$ & 268.10 & 8.3 & 0.1 & 69 \\
\hline $30-1,50$ & 276.00 & 5.6 & 0.2 & 45 \\
\hline $30-2,50$ & 277.50 & 4.9 & 0.1 & 40 \\
\hline $30-3,50$ & 279.00 & 4.5 & 0.1 & 37 \\
\hline $30-4,50$ & 280.50 & 8.1 & 0.1 & 66 \\
\hline $30-5,50$ & 282.00 & 8.7 & 0.1 & 72 \\
\hline $31-1,50$ & 285.50 & 4.0 & 0.1 & 33 \\
\hline $31-2,50$ & 287.00 & 4.2 & 0.1 & 34 \\
\hline $31-3,50$ & 288.50 & 6.6 & 0.1 & 54 \\
\hline $31-4,50$ & 290.00 & 6.6 & 0.1 & 54 \\
\hline $31-5,50$ & 29.50 & 7.2 & 0.1 & 59 \\
\hline $31-6,50$ & 293.00 & 6.2 & 0.1 & 51 \\
\hline $32-1,50$ & 295.00 & 5.0 & 0.1 & 41 \\
\hline $32-2,50$ & 296.50 & 4.6 & 0.1 & 37 \\
\hline $32-3,50$ & 298.00 & 3.9 & 0.1 & 31 \\
\hline $32-4,50$ & 299.50 & 3.1 & 0.1 & 25 \\
\hline $32-5,50$ & 301.00 & 3.1 & 0.1 & 25 \\
\hline $32-6,50$ & 302.50 & 2.9 & 0.1 & 24 \\
\hline $33-1,50$ & 304.50 & 3.4 & 0.2 & 27 \\
\hline $33-2,50$ & 306.00 & 3.0 & 0.1 & 24 \\
\hline $33-4,50$ & 307.50 & 3.1 & 0.1 & 25 \\
\hline $33-4,50$ & 309.00 & 2.2 & 0.1 & 18 \\
\hline $33-5,50$ & 310.50 & 2.3 & 0.1 & 18 \\
\hline $33-6,50$ & 312.00 & 3.0 & 0.1 & 24 \\
\hline $34-1,50$ & 314.00 & 5.3 & 0.1 & 44 \\
\hline $34-2,50$ & 315.50 & 61 & 0.1 & 50 \\
\hline $34-3,50$ & 317.00 & 5.9 & 0.1 & 48 \\
\hline $34-4,50$ & 318.50 & 6.3 & 0.1 & 52 \\
\hline $34-5,50$ & 320.00 & 3.9 & 0.1 & 31 \\
\hline \multicolumn{5}{|l|}{ Hole 409} \\
\hline $2-1,50$ & 25.00 & 2.5 & 0.1 & 20 \\
\hline $2-2,50$ & 26.50 & 7.2 & 0.1 & 59 \\
\hline $2-3,50$ & 28.00 & 5.1 & 0.1 & 42 \\
\hline $4-1,50$ & 44.00 & 1.1 & 0.7 & 3 \\
\hline $4-2,50$ & 45.50 & 1.4 & 0.2 & 9 \\
\hline $4-3,50$ & 47.00 & 1.5 & 0.1 & 11 \\
\hline $4-4,50$ & 48.50 & 4.8 & 0.1 & 39 \\
\hline $4-5,19$ & 49.69 & 1.4 & 0.1 & 11 \\
\hline $5-1,50$ & 53.50 & 2.9 & 0.1 & 23 \\
\hline $5-2,50$ & 55.00 & 3.4 & 0.2 & 26 \\
\hline $5-3,50$ & 56.50 & 4.4 & 0.1 & 36 \\
\hline $5-4,60$ & 58.10 & 8.9 & 0.2 & 72 \\
\hline $6-1,64$ & 63.14 & 2.1 & 0.3 & 15 \\
\hline $6-2,76$ & 64.76 & 3.3 & 0.2 & 26 \\
\hline $6-3,67$ & 66.17 & 1.4 & 0.1 & 11 \\
\hline $7-1,75$ & 72.75 & 2.5 & 0.1 & 20 \\
\hline $7-2,20$ & 73.70 & 2.9 & 0.1 & 23 \\
\hline $7-3,60$ & 75.60 & 2.4 & 0.2 & 18 \\
\hline
\end{tabular}


TABLE 2 - Continued

\begin{tabular}{|c|c|c|c|c|}
\hline $\begin{array}{c}\text { Sample } \\
\text { (Interval in cm) }\end{array}$ & $\begin{array}{l}\text { Depth } \\
\text { (m) }\end{array}$ & $\begin{array}{c}\text { Total } \\
\text { Carbon (\%) }\end{array}$ & $\begin{array}{c}\text { Organic } \\
\text { Carbon (\%) }\end{array}$ & $\mathrm{CaCO}_{3}$ \\
\hline $7-5,35$ & 78.35 & 3.6 & 0.1 & 29 \\
\hline $7-6,47$ & 79.97 & 4.6 & 0.1 & 37 \\
\hline \multicolumn{5}{|l|}{ Hole 410} \\
\hline $1-1,89$ & 0.89 & 10.8 & 0.1 & 89 \\
\hline $1-2,31$ & 1.81 & 5.5 & 0.1 & 45 \\
\hline $1-3,31$ & 3.31 & 10.5 & 0.1 & 87 \\
\hline $1-4,46$ & 4.96 & 6.6 & 0.2 & 54 \\
\hline $1-5,43$ & 6.43 & 8.3 & 0.2 & 67 \\
\hline $2-1,63$ & 8.13 & 10.7 & 0.1 & 89 \\
\hline $2-2,45$ & 9.45 & 5.9 & 0.2 & 48 \\
\hline $2-3,99$ & 11.49 & 10.9 & 0.1 & 90 \\
\hline $2-4,33$ & 12.33 & 11.0 & 0.1 & 91 \\
\hline $3-1,125$ & 18.25 & 11.3 & 0.1 & 93 \\
\hline $3-2,36$ & 18.86 & 11.5 & 0.1 & 95 \\
\hline $3-3,34$ & 20.34 & 10.7 & 0.1 & 89 \\
\hline $3-4,35$ & 21.85 & 10.3 & 0.1 & 85 \\
\hline $4-1,35$ & 26.85 & 10.9 & 0.1 & 90 \\
\hline $4-2,49$ & 28.49 & 1.7 & 0.2 & 12 \\
\hline $4-3,43$ & 29.93 & 9.1 & 0.1 & 75 \\
\hline $4-4,46$ & 31.46 & 6.7 & 0.2 & 54 \\
\hline $5-1,65$ & 36.65 & 7.6 & 0.2 & 62 \\
\hline $5-2,63$ & 38.13 & 10.2 & 0.1 & 84 \\
\hline $5-3,66$ & 39.66 & 10.4 & 0.1 & 86 \\
\hline $5-4,75$ & 41.25 & 10.7 & 0.1 & 89 \\
\hline $5-5,35$ & 42.35 & 6.3 & 0.2 & 51 \\
\hline $5-6,85$ & 44.35 & 6.1 & 0.2 & 50 \\
\hline $6-1,85$ & 46.35 & 9.8 & 0.2 & 81 \\
\hline $6-2,36$ & 47.36 & 9.9 & 0.2 & 81 \\
\hline $6-3,45$ & 48.95 & 8.8 & 0.2 & 72 \\
\hline $7-1,33$ & 55.33 & 9.4 & 0.2 & 77 \\
\hline $7-2,16$ & 56.66 & 7.6 & 0.2 & 62 \\
\hline $8-1,46$ & 64.96 & 9.4 & 0.2 & 78 \\
\hline $8-1,50$ & 65.00 & 9.4 & 0.2 & 77 \\
\hline $8-2,46$ & 66.46 & 10.7 & 0.1 & 89 \\
\hline $8-3,46$ & 67.96 & 9.4 & 0.1 & 77 \\
\hline $8-4,76$ & 69.76 & 10.4 & 0.1 & 86 \\
\hline $8-5,46$ & 70.96 & 11.1 & 0.6 & 87 \\
\hline $8-6,50$ & 72.50 & 9.8 & 0.1 & 81 \\
\hline $10-1,50$ & 84.00 & 9.3 & 0.1 & 77 \\
\hline $10-2,50$ & 85.50 & 10.2 & 0.1 & 84 \\
\hline $10-3,50$ & 87.00 & 10.3 & 0.1 & 85 \\
\hline $10-4,50$ & 88.50 & 7.7 & 0.2 & 62 \\
\hline $10-5,50$ & 90.00 & 8.3 & 0.1 & 68 \\
\hline $10-6,50$ & 91.50 & 8.8 & 0.1 & 72 \\
\hline $10-7,20$ & 92.70 & 10.9 & 0.1 & 90 \\
\hline $11-1,50$ & 93.50 & 10.6 & 0.1 & 87 \\
\hline $11-2,50$ & 95.00 & 10.1 & 0.1 & 83 \\
\hline $11-3,50$ & 96.50 & 10.5 & 0.1 & 87 \\
\hline $11-4,50$ & 98.00 & 10.7 & 0.1 & 89 \\
\hline $11-5,50$ & 99.50 & 10.5 & 0.1 & 86 \\
\hline $11-6,50$ & 101.00 & 10.6 & 0.1 & 88 \\
\hline $11-7,20$ & 102.20 & 11.1 & 0.1 & 92 \\
\hline $13-1,50$ & 112.50 & 10.7 & 0.1 & 88 \\
\hline $13-2,50$ & 114.00 & 10.2 & 0.1 & 84 \\
\hline $14-1,50$ & 122.00 & 10.0 & 0.1 & 83 \\
\hline $14-1,53$ & 122.03 & 9.8 & 0.1 & 81 \\
\hline $14-2,50$ & 123.50 & 9.9 & 0.1 & 82 \\
\hline $14-2,53$ & 123.53 & 9.8 & 0.1 & 80 \\
\hline $14-3,50$ & 125.00 & 8.6 & 0.1 & 71 \\
\hline $14-3,53$ & 125.03 & 8.6 & 0.2 & 71 \\
\hline $14-4,50$ & 126.50 & 8.1 & 0.2 & 66 \\
\hline $14-4,53$ & 126.53 & 8.3 & 0.2 & 68 \\
\hline $14-5,50$ & 128.00 & 11.4 & 0.1 & 94 \\
\hline $14-5,53$ & 128.03 & 11.3 & 0.1 & 94 \\
\hline $14-6,50$ & 129.50 & 11.1 & 0.1 & 92 \\
\hline $14-6,53$ & 129.53 & 11.2 & 0.1 & 93 \\
\hline $14-7,20$ & 130.70 & 9.6 & 0.2 & 78 \\
\hline
\end{tabular}

TABLE 2 - Continued

\begin{tabular}{|c|c|c|c|c|}
\hline $\begin{array}{c}\text { Sample } \\
\text { (Interval in } \mathrm{cm} \text { ) }\end{array}$ & $\begin{array}{l}\text { Depth } \\
\text { (m) }\end{array}$ & $\begin{array}{c}\text { Total } \\
\text { Carbon (\%) }\end{array}$ & $\begin{array}{c}\text { Organic } \\
\text { Carbon (\%) }\end{array}$ & $\mathrm{CaCO}_{3}$ \\
\hline $14-7,23$ & 130.73 & 10.0 & 0.2 & 82 \\
\hline $15-1,50$ & 131.50 & 10.5 & 0.1 & 87 \\
\hline $15-1,53$ & 131.53 & 10.5 & 0.1 & 87 \\
\hline $15-2,80$ & 133.30 & 10.7 & 0.1 & 89 \\
\hline $15-2,83$ & 133.33 & 10.7 & 0.1 & 89 \\
\hline $15-3,80$ & 134.80 & 10.9 & 0.1 & 90 \\
\hline $15-3,84$ & 134.84 & 10.9 & 0.1 & 90 \\
\hline $15-4,50$ & 136.00 & 11.0 & 0.1 & 91 \\
\hline $15-4,53$ & 136.03 & 11.1 & 0.1 & 92 \\
\hline $15-5,50$ & 137.50 & 11.2 & 0.1 & 93 \\
\hline $15-5,53$ & 137.53 & 11.2 & 0.1 & 93 \\
\hline $15-6,50$ & 139.00 & 10.7 & 0.1 & 89 \\
\hline $15-6,53$ & 139.03 & 10.8 & 0.1 & 89 \\
\hline $16-1,50$ & 141.00 & 10.9 & 0.1 & 90 \\
\hline $16-1,53$ & 141.03 & 11.0 & 0.1 & 91 \\
\hline $16-2,50$ & 142.50 & 11.0 & 0.1 & 91 \\
\hline $16-2,53$ & 142.53 & 10.9 & 0.1 & 89 \\
\hline $16-3,50$ & 144.00 & 10.8 & 0.1 & 89 \\
\hline $16-3,53$ & 144.03 & 10.8 & 0.1 & 89 \\
\hline $16-4,50$ & 145.50 & 10.8 & 0.1 & 89 \\
\hline $16-4,53$ & 145.53 & 10.9 & 0.1 & 90 \\
\hline $16-5,50$ & 147.00 & 10.8 & 0.1 & 90 \\
\hline $16-5,53$ & 147.03 & 10.8 & 0.1 & 90 \\
\hline $16-6,50$ & 148.50 & 10.7 & 0.1 & 89 \\
\hline $16-6,53$ & 148.53 & 10.7 & 0.1 & 89 \\
\hline $18-1,84$ & 160.34 & 11.4 & 0.1 & 95 \\
\hline $18-1,87$ & 160.37 & 11.3 & 0.1 & 94 \\
\hline $18-2,84$ & 161.84 & 11.3 & 0.1 & 94 \\
\hline $18-2,86$ & 161.86 & 11.4 & 0.1 & 95 \\
\hline $18-3,74$ & 163.24 & 11.4 & 0.1 & 95 \\
\hline $18-3,77$ & 163.27 & 11.4 & 0.1 & 95 \\
\hline $18-4,55$ & 164.55 & 11.4 & 0.1 & 95 \\
\hline $18-4,58$ & 164.58 & 11.4 & 0.1 & 94 \\
\hline $18-5,44$ & 165.94 & 11.5 & 0.1 & 96 \\
\hline $18-5,47$ & 165.97 & 11.5 & 0.1 & 95 \\
\hline $19-1,97$ & 169.97 & 11.5 & 0.1 & 95 \\
\hline $19-1,100$ & $170 ? 00$ & 11.4 & 0.1 & 95 \\
\hline $19-2,54$ & 171.04 & 11.5 & 0.1 & 95 \\
\hline $19-3,60$ & 172.60 & 11.4 & 0.1 & 94 \\
\hline $19-4,80$ & 174.30 & 11.0 & 0.1 & 91 \\
\hline $19-5,50$ & 175.50 & 11.4 & 0.1 & 94 \\
\hline $19-6,60$ & 177.10 & 11.5 & 0.1 & 96 \\
\hline $20-1,58$ & 179.08 & 11.5 & 0.1 & 95 \\
\hline $20-2,59$ & 180.59 & 11.5 & 0.1 & 96 \\
\hline $20-3,8$ & 181.58 & 11.5 & 0.1 & 95 \\
\hline $21-1,70$ & 188.70 & 11.5 & 0.1 & 95 \\
\hline $21-2,90$ & 190.40 & 11.5 & 0.1 & 95 \\
\hline $21-3,80$ & 191.80 & 11.6 & 0.1 & 96 \\
\hline $21-4,60$ & 193.10 & 11.3 & 0.1 & 94 \\
\hline $21-5,70$ & 194.70 & 11.5 & 0.1 & 95 \\
\hline $21-6,70$ & 196.20 & 11.6 & 0.1 & 96 \\
\hline $21-7,15$ & 197.15 & 11.6 & 0.1 & 96 \\
\hline $22-1,80$ & 198.30 & 11.4 & 0.1 & 95 \\
\hline $22-1,90$ & 198.40 & 11.4 & 0.1 & 95 \\
\hline $22-2,90$ & 199.90 & 11.4 & 0.1 & 95 \\
\hline $22-2,106$ & 200.06 & 11.4 & 0.1 & 94 \\
\hline $22-3,50$ & 201.00 & 11.4 & 0.1 & 94 \\
\hline $22-3,60$ & 201.10 & 11.4 & 0.1 & 94 \\
\hline $22-4,40$ & 202.40 & 11.4 & 0.1 & 94 \\
\hline $22-4,44$ & 202.44 & 11.4 & 0.1 & 94 \\
\hline $22-5,75$ & 204.25 & 11.2 & 0.1 & 93 \\
\hline $22-6,65$ & 205.65 & 11.5 & 0.1 & 95 \\
\hline $22-6,67$ & 205.67 & 11.4 & 0.1 & 94 \\
\hline $22-7,19$ & 206.69 & 11.3 & 0.1 & 94 \\
\hline $22-7,21$ & 206.71 & 11.4 & 0.1 & 95 \\
\hline $23-1,70$ & 207.70 & 11.3 & 0.1 & 94 \\
\hline $23-1,79$ & 207.79 & 11.3 & 0.1 & 94 \\
\hline $23-2,103$ & 209.53 & 11.3 & 0.1 & 93 \\
\hline
\end{tabular}


TABLE 2 - Continued

\begin{tabular}{|c|c|c|c|c|}
\hline $\begin{array}{c}\text { Sample } \\
\text { (Interval in } \mathrm{cm} \text { ) }\end{array}$ & $\begin{array}{l}\text { Depth } \\
\text { (m) }\end{array}$ & $\begin{array}{c}\text { Total } \\
\text { Carbon }(\%)\end{array}$ & $\begin{array}{c}\text { Organic } \\
\text { Carbon (\%) }\end{array}$ & $\mathrm{CaCO}_{3}$ \\
\hline $23-2,106$ & 209.56 & 11.3 & 0.1 & 94 \\
\hline $23-3,87$ & 210.87 & 11.1 & 0.1 & 92 \\
\hline $23-3,90$ & 210.90 & 11.4 & 0.1 & 95 \\
\hline $23-4,80$ & 212.30 & 11.5 & 0.1 & 95 \\
\hline $23-4,84$ & 212.34 & 11.5 & 0.1 & 95 \\
\hline $23-5,75$ & 213.75 & 11.5 & 0.1 & 95 \\
\hline $23-6,50$ & 215.00 & 11.6 & 0.1 & 96 \\
\hline $23-6,55$ & 215.05 & 11.4 & 0.1 & 94 \\
\hline $24-1,60$ & 217.10 & 11.4 & 0.1 & 95 \\
\hline $24-1,64$ & 217.14 & 11.4 & 0.1 & 95 \\
\hline $24-2,80$ & 218.80 & 11.4 & 0.1 & 94 \\
\hline $24-2,83$ & 218.83 & 11.4 & 0.1 & 94 \\
\hline $24-3,60$ & 220.10 & 11.5 & 0.1 & 96 \\
\hline $24-3,64$ & 220.14 & 11.5 & 0.1 & 95 \\
\hline $24-4,40$ & 221.40 & 11.5 & 0.1 & 96 \\
\hline $24-4,43$ & 221.43 & 11.5 & 0.1 & 95 \\
\hline $24-5,70$ & 223.20 & 11.5 & 0.1 & 96 \\
\hline $24-6,52$ & 224.52 & 11.5 & 0.1 & 95 \\
\hline $24-6,55$ & 224.55 & 11.4 & 0.1 & 95 \\
\hline $24-7,10$ & 225.60 & 11.3 & 0.1 & 94 \\
\hline $24-7,13$ & 225.63 & 11.3 & 0.1 & 94 \\
\hline $25-1,70$ & 226.70 & 11.3 & 0.1 & 94 \\
\hline $25-1,74$ & 226.74 & 11.3 & 0.1 & 94 \\
\hline $25-2,50$ & 228.00 & 11.2 & 0.1 & 93 \\
\hline $25-2,53$ & 228.03 & 11.2 & 0.1 & 93 \\
\hline $25-3,60$ & 229.60 & 11.4 & 0.1 & 94 \\
\hline $25-3,65$ & 229.65 & 11.4 & 0.1 & 94 \\
\hline $25-4,50$ & 231.00 & 11.3 & 0.1 & 94 \\
\hline $25-4,53$ & 231.03 & 11.3 & 0.1 & 94 \\
\hline $25-5,50$ & 232.50 & 11.5 & 0.1 & 95 \\
\hline $25-6,50$ & 234.00 & 11.3 & 0.1 & 94 \\
\hline $25-6,53$ & 234.03 & 11.4 & 0.1 & 94 \\
\hline $25-7,20$ & 235.20 & 11.4 & 0.1 & 94 \\
\hline $25-7,23$ & 235.23 & 11.3 & 0.1 & 93 \\
\hline $26-1,30$ & 235.80 & 11.2 & 0.1 & 92 \\
\hline $26-1,33$ & 235.83 & 11.2 & 0.1 & 93 \\
\hline $26-2,30$ & 237.30 & 11.2 & 0.1 & 93 \\
\hline $26-3,30$ & 238.80 & 11.2 & 0.1 & 92 \\
\hline $26-3,33$ & 238.83 & 11.2 & 0.1 & 93 \\
\hline $27-1,50$ & 245.50 & 11.2 & 0.1 & 92 \\
\hline $27-1,53$ & 245.53 & 11.2 & 0.1 & 93 \\
\hline $27-2,50$ & 247.00 & 11.1 & 0.1 & 92 \\
\hline $27-3,53$ & 248.53 & 11.3 & 0.1 & 93 \\
\hline $27-3,50$ & 248.50 & 11.2 & 0.1 & 93 \\
\hline $28-1,50$ & 255.00 & 11.0 & 0.1 & 91 \\
\hline $28-1,53$ & 255.03 & 10.9 & 0.1 & 90 \\
\hline $28-3,50$ & 256.50 & 11.1 & 0.1 & 92 \\
\hline $28-2,53$ & 256.53 & 10.9 & 0.1 & 90 \\
\hline $28-3,50$ & 258.00 & 11.0 & 0.1 & 91 \\
\hline $28-4,50$ & 259.50 & 11.2 & 0.1 & 93 \\
\hline $28-4,53$ & 259.53 & 11.2 & 0.1 & 92 \\
\hline $29-1,50$ & 264.50 & 11.3 & 0.1 & 94 \\
\hline $29-1,53$ & 264.53 & 11.3 & 0.1 & 93 \\
\hline $29-2,50$ & 266.00 & 11.2 & 0.1 & 92 \\
\hline $29-2,53$ & 266.03 & 11.1 & 0.1 & 91 \\
\hline $29-3,50$ & 267.50 & 11.0 & 0.1 & 91 \\
\hline $29-4,50$ & 269.00 & 11.0 & 0.1 & 91 \\
\hline $29-4,53$ & 269.03 & 11.1 & 0.1 & 91 \\
\hline $29-5,20$ & 270.20 & 11.2 & 0.1 & 92 \\
\hline $29-5,23$ & 270.23 & 11.2 & 0.1 & 93 \\
\hline $30-1,50$ & 274.00 & 11.2 & 0.1 & 92 \\
\hline $30-1,53$ & 274.03 & 11.2 & 0.1 & 93 \\
\hline $30-2,50$ & 275.50 & 11.2 & 0.1 & 93 \\
\hline $30-2,53$ & 275.53 & 11.2 & 0.1 & 93 \\
\hline $30-3,50$ & 277.00 & 11.1 & 0.1 & 92 \\
\hline $30-3,53$ & 277.03 & 11.0 & 0.1 & 91 \\
\hline $30-4,50$ & 278.50 & 11.3 & 0.1 & 93 \\
\hline $30-5,50$ & 280.00 & 11.0 & 0.1 & 91 \\
\hline
\end{tabular}

TABLE 2 - Continued

\begin{tabular}{|c|c|c|c|c|}
\hline $\begin{array}{c}\text { Sample } \\
\text { (Interval in } \mathrm{cm} \text { ) }\end{array}$ & $\begin{array}{l}\text { Depth } \\
(\mathrm{m})\end{array}$ & $\begin{array}{c}\text { Total } \\
\text { Carbon }(\%)\end{array}$ & $\begin{array}{c}\text { Organic } \\
\text { Carbon (\%) }\end{array}$ & $\mathrm{CaCO}_{3}$ \\
\hline $30-5,53$ & 280.03 & 11.2 & 0.1 & 93 \\
\hline $30-6,50$ & 281.50 & 11.3 & 0.1 & 94 \\
\hline $30-6,53$ & 281.53 & 11.3 & 0.1 & 94 \\
\hline $30-7,20$ & 282.70 & 11.3 & 0.1 & 94 \\
\hline $30-7,23$ & 282.73 & 11.3 & 0.1 & 93 \\
\hline $31-1,88$ & 283.88 & 11.2 & 0.1 & 93 \\
\hline $31-1,90$ & 283.90 & 11.3 & 0.1 & 93 \\
\hline $31-2,79$ & 285.29 & 11.3 & 0.1 & 93 \\
\hline $31-3,65$ & 286.65 & 11.1 & 0.1 & 92 \\
\hline $31-3,68$ & 286.68 & 11.2 & 0.1 & 93 \\
\hline 314,35 & 287.85 & 11.2 & 0.1 & 92 \\
\hline $31-4,37$ & 287.87 & 11.1 & 0.1 & 92 \\
\hline $32-1,65$ & 293.15 & 11.2 & 0.1 & 93 \\
\hline $32-1,57$ & 293.17 & 11.2 & 0.1 & 93 \\
\hline $32-2,54$ & 294.54 & 11.2 & 0.1 & 92 \\
\hline $32-3,82$ & 296.32 & 11.1 & 0.1 & 92 \\
\hline $32-3,84$ & 296.34 & 11.2 & 0.1 & 92 \\
\hline $33-1,71$ & 302.71 & 11.2 & 0.1 & 92 \\
\hline $33-2,40$ & 303.90 & 1.8 & 0.1 & 15 \\
\hline $33-2,41$ & 303.91 & 11.1 & 0.1 & 92 \\
\hline $34-1,17$ & 311.67 & 11.0 & 0.1 & 91 \\
\hline $34-2,78$ & 313.78 & 11.2 & 0.0 & 93 \\
\hline $34-2,79$ & 313.79 & 11.4 & 0.1 & 94 \\
\hline $35-1,76$ & 321.76 & 11.3 & 0.1 & 94 \\
\hline $35-1,77$ & 321.77 & 11.4 & 0.1 & 95 \\
\hline $35-2,77$ & 323.27 & 11.3 & 0.1 & 94 \\
\hline $35-3,66$ & 324.66 & 11.5 & 0.1 & 95 \\
\hline $35-3,67$ & 324.67 & 11.4 & 0.1 & 95 \\
\hline $36-1,75$ & 331.25 & 10.9 & 0.1 & 90 \\
\hline $36-1,78$ & 331.28 & 11.0 & 0.1 & 91 \\
\hline $36-2,135$ & 333.35 & 11.0 & 0.1 & 91 \\
\hline $36-3,47$ & 333.97 & 10.7 & 0.1 & 89 \\
\hline $36-3,52$ & 334.02 & 10.8 & 0.1 & 90 \\
\hline
\end{tabular}

Hole 410A

$\begin{array}{rrrrr}1-1,74 & 325.74 & 5.0 & 0.2 & 40 \\ 1-1,76 & 325.76 & 5.0 & 0.2 & 40 \\ 1-2,94 & 327.44 & 10.7 & 0.1 & 88 \\ 1-2,97 & 327.47 & 10.6 & 0.1 & 88 \\ 1-3,64 & 328.64 & 10.8 & 0.1 & 89 \\ 1-3,67 & 328.67 & 10.7 & 0.1 & 88 \\ 1-4,73 & 330.23 & 10.8 & 0.1 & 89 \\ 1-4,75 & 330.25 & 10.7 & 0.1 & 88 \\ 1-5,90 & 331.90 & 11.4 & 0.1 & 94 \\ 1-5,93 & 331.93 & 11.2 & 0.1 & 93 \\ 1-6,22 & 332.72 & 10.9 & 0.1 & 90 \\ 1-6,25 & 332.75 & 10.8 & 0.1 & 90\end{array}$

Hole 411

$\begin{array}{lllll}1, \mathrm{CC} & 74.70 & 10.7 & 0.1 & 89 \\ 1-2,33 & 75.83 & 11.1 & 0.0 & 92 \\ 1-4,75 & 79.25 & 11.3 & 0.0 & 94\end{array}$

Hole 412

$1-1,50$
$1-1,142$
$1-2,50$
$2-1,50$
$2-2,50$
$2-3,50$
$2-4,50$
$2-5,50$
$2-6,60$
$3-1,50$
$3-2,50$
$3-3,50$
$3-4,30$

$\begin{array}{rr}0.50 & 10.4 \\ 1.42 & 10.1 \\ 2.0 & 9.3 \\ 4.50 & 10.0 \\ 6.00 & 9.0 \\ 7.50 & 9.3 \\ 9.00 & 10.3 \\ 10.50 & 10.0 \\ 12.10 & 10.7 \\ 14.00 & 10.8 \\ 15.50 & 9.7 \\ 17.00 & 10.0 \\ 18.30 & 8.6\end{array}$

\begin{tabular}{ll}
0.1 & 86 \\
0.2 & 82 \\
0.3 & 74 \\
0.1 & 82 \\
0.3 & 73 \\
0.3 & 75 \\
0.1 & 84 \\
0.2 & 82 \\
0.1 & 88 \\
0.1 & 89 \\
0.1 & 80 \\
0.1 & 83 \\
0.2 & 70 \\
\hline
\end{tabular}


TABLE 2 - Continued

\begin{tabular}{lrccc}
\hline $\begin{array}{c}\text { Sample } \\
\text { (Interval in cm) }\end{array}$ & $\begin{array}{c}\text { Depth } \\
(\mathrm{m})\end{array}$ & $\begin{array}{c}\text { Total } \\
\text { Carbon }(\%)\end{array}$ & $\begin{array}{c}\text { Organic } \\
\text { Carbon }(\%)\end{array}$ & $\mathrm{CaCO}_{3}$ \\
\hline $3-5,60$ & 20.10 & 9.8 & 0.1 & 81 \\
$3-6,50$ & 21.50 & 9.9 & 0.2 & 81 \\
$4-1,50$ & 23.50 & 8.0 & 0.3 & 64 \\
$4-2,50$ & 25.00 & 9.8 & 0.2 & 80 \\
$4-3,50$ & 26.50 & 10.5 & 0.1 & 87 \\
$4-4,50$ & 28.00 & 10.2 & 0.1 & 84 \\
$4-5,50$ & 29.50 & 10.5 & 0.2 & 85 \\
$4-6,50$ & 31.00 & 10.7 & 0.1 & 88 \\
$5-1,50$ & 52.00 & 10.6 & 0.2 & 86 \\
$5-2,50$ & 53.50 & 10.5 & 0.2 & 86 \\
$5-3,50$ & 55.00 & 9.7 & 0.2 & 79 \\
$5-4,50$ & 56.50 & 11.4 & 0.1 & 94 \\
$6-1,50$ & 80.50 & 10.8 & 0.1 & 89 \\
$6-2,50$ & 82.00 & 10.2 & 0.1 & 84 \\
$6-3,50$ & 83.50 & 9.4 & 0.3 & 76 \\
$6-4,30$ & 94.80 & 11.1 & 0.1 & 91 \\
$7-1,50$ & 90.00 & 11.2 & 0.1 & 92 \\
$7-2,50$ & 91.50 & 10.6 & 0.1 & 88 \\
$8-1,76$ & 99.76 & 10.1 & 0.2 & 83 \\
$8-2,76$ & 101.26 & 10.4 & 0.1 & 86 \\
$8-3,76$ & 102.76 & 10.5 & 0.1 & 86 \\
$8-4,76$ & 104.26 & 10.2 & 0.1 & 84 \\
$8-5,76$ & 105.76 & 10.9 & 0.1 & 89 \\
$8-6,76$ & 107.26 & 11.0 & 0.1 & 91 \\
& & & & \\
\hline
\end{tabular}

TABLE 2 - Continued

\begin{tabular}{lcccc}
\hline $\begin{array}{c}\text { Sample } \\
\text { (Interval in cm) }\end{array}$ & $\begin{array}{c}\text { Depth } \\
(\mathrm{m})\end{array}$ & $\begin{array}{c}\text { Total } \\
\text { Carbon }(\%)\end{array}$ & $\begin{array}{c}\text { Organic } \\
\text { Carbon }(\%)\end{array}$ & $\mathrm{CaCO}_{3}$ \\
\hline $8-7,18$ & 108.18 & 11.0 & 0.1 & 90 \\
$9-1,103$ & 109.53 & 10.9 & 0.1 & 90 \\
$9-2,80$ & 110.80 & 10.5 & 0.1 & 86 \\
$9-3,80$ & 112.30 & 10.7 & 0.1 & 88 \\
$9-4,80$ & 113.80 & 11.0 & 0.1 & 91 \\
$9-5,80$ & 115.30 & 11.4 & 0.1 & 94 \\
$9-6,60$ & 116.60 & 11.1 & 0.1 & 91 \\
$10-1,80$ & 118.80 & 11.0 & 0.1 & 90 \\
$10-2,80$ & 120.30 & 11.1 & 0.1 & 91 \\
$10-3,110$ & 122.10 & 11.0 & 0.1 & 91 \\
$10-4,80$ & 123.30 & 11.1 & 0.1 & 91 \\
$10-5,20$ & 124.20 & 11.0 & 0.1 & 91 \\
$10-6,30$ & 125.80 & 11.2 & 0.1 & 92 \\
$12-1,80$ & 137.80 & 10.9 & 0.1 & 90 \\
$12-2,80$ & 139.30 & 10.9 & 0.1 & 90 \\
$12-3,20$ & 140.20 & 10.9 & 0.1 & 90 \\
$13-1,19$ & 146.69 & 11.2 & 0.1 & 92 \\
$14-1,79$ & 156.79 & 10.7 & 0.1 & 88 \\
$14-2,80$ & 158.30 & 10.4 & 0.1 & 86 \\
$14-3,80$ & 159.80 & 10.2 & 0.1 & 84 \\
$14-4,60$ & 161.10 & 10.2 & 0.1 & 84 \\
$14-5,80$ & 162.80 & 10.0 & 0.1 & 83 \\
$14-6,80$ & 164.30 & 11.1 & 0.1 & 91 \\
$14-7,15$ & 165.15 & 12.1 & 0.1 & 100 \\
& & & & \\
\hline & & & &
\end{tabular}

